\title{
Methods for Panoramic Visualization and Digital Analysis of Thermophysical Flow Fields. A Review.
}

\author{
I.A. Znamenskaya ${ }^{1}$ \\ Lomonosov Moscow State University \\ ${ }^{1}$ ORCID: oooo-0oo1-6362-9496, znamen@phys.msu.ru
}

\begin{abstract}
The paper presents a review of modern methods in the registration, processing, and analysis of dynamic processes in liquids, gases, plasmas, multiphase media, which are realized in researches and technology. The review author observes both the physical fundamentals of flow visualization and the basics of modern technologies for digital processing of recorded flow images. A brief analysis of the panoramic visualization methods progress history covers a period of one and a half centuries. In the works of the last decade, the focus is on the methods of computer processing, tools, technologies for analyzing and recognizing of panoramic thermophysical fields, which make it possible to obtain quantitative information about flows. The review contains an analysis of publications describing the main modern methods of visualization of flows: methods based on the phenomenon of refraction; electroluminescence; on digital tracing (particle image velocimetry), visualization of surface flows (PSP and TSP coatings, liquid crystals; oil coatings). Particular attention is paid to methods using crosscorrelation image processing algorithms. Those are: digital tracing (PIV), shadow background method (in English Background Oriented Schlieren - BOS), seedless shadow methods, thermographic PIV, velocity measurement in viscous coatings, micro, tomographic modifications of PIV, etc. The actual problem of digital data analyzing in a panoramic experiment is touched upon - the problem of big data. Examples of the machine learning use in the analysis of big data sets of shadow surveys are given. Some examples of numerical simulation data visualization (simulation of experimental flowfields) are considered.

Keywords: panoramic visualization, digital image analysis, optical methods, velocity field, density gradient, discontinuity recognition, PIV, BOS methods, computer processing.
\end{abstract}

\section{Introduction}

Flow visualization in environment and in technical devices as a way of recording and transmitting information about various dynamic processes has existed for several centuries. Understanding of complex non-stationary processes occurring in the flow of gases, liquids, plasmas is possible by observing the complete flow configuration. In this respect, panoramic visualization of the flow field is the most important way to obtain information about such flows in experimental studies in thermal physics and mechanics of gas, liquid and plasma.

It is generally accepted that scientific visualization of flows appeared in the Renaissance dynamic processes and phenomena of fluid mechanics and physics were recorded in graphic images. The study of gas and liquid flows helped the genius of the Italian Renaissance Leonardo da Vinci to think over projects for creating vehicles by air, water, and land. Since the Renaissance, visualization has developed mainly in two forms - as a fine art and as a science. The newly discovered physical principles of image registration were used simultaneously in a new level of scientific visualization and in art. For example, the invention of photography was originally used primarily in everyday life; it was only at the end of the 19th century that scientific photography appeared. In 1839 in Paris, at a meeting with the participation of the Academy of Sciences 
members, it was announced that Daguerre had discovered a way to develop and fix photographic images. After this event, the development of scientific visualization began.

Panoramic visualization of thermodynamic fields includes two-dimensional, threedimensional, four-dimensional presentation of the results of measurements and digital analysis of the tested fields of gas-dynamic, thermodynamic parameters, as well as the results of numerical calculations of dynamic processes. Panoramic visualization of flows is in demand in many natural sciences - thermal physics, mechanics, geophysics, medicine, plasma physics, biology, psychology, etc.

A. Tepler was the first to propose the so-called sweep method (a kind of shadow method) for studying optical inhomogeneities in a transparent medium and shock wave from an electric spark was observed (1867). E. Mach proposed several flow visualization techniques. In the 8os of the 19th century, he received the first shadow image of a bow shock wave in front of a bullet flying at supersonic speed, and later he received flow interferograms [1]. French naturalist J.E. Marey was the first to use multi-frame photography to record flow movements and, having built a wind tunnel, visualized a smoke system around obstacles and the transition to turbulence. [2].

Over the past two centuries, the development of panoramic visualization of thermodynamic, hydrodynamic fields was mainly determined by the following five factors:

1. Progress in the creation of registration materials;

2. Progress in the creation of equipment for recording images;

3. Creation of new sources of generation (and transmission) of probing electromagnetic radiation;

4. Development of visual information storage methods.

5. Development of digital methods of visualization data analysis and processing (beginning from the 8oth of the 2oth century).

Registration materials have progressed from solid carriers (paper, photographic plates, photographic films) to digital matrices. Recording equipment, respectively - from direct observation with the eyes, then observation using pinhole cameras, optics. From the middle of the 19th century, photographic equipment began to be used for scientific visualization, then film cameras, video cameras, and high-speed drum cameras. In the middle of the 2oth century, electrooptical devices appeared. Light sources are also constantly developing: from - solar radiation, candles, electric discharges, lasers, cumulative sources, LED sources, etc. Modern sources emit in a wide range of wavelengths and pulse durations.

In the new digital period, there is a constant renewal of technologies for the manufacture of specialized cameras for registering processes in flows; there is a competition between companies for increasing the space-time, spectral characteristics of cameras.

Methods for visualizing the structure and flows' parameters are based on the physical properties of electromagnetic radiation and its interactions with media, including various physical processes:

- Scattering;

- Refraction;

- Absorption

- Reflection

- Interference

- Dispersion

- Luminescence

- Effects of nonlinear optics

The listed processes include the light properties, which can be interpreted within the framework of geometric, wave, quantum models. Nowadays, for the competent use of experimental visualization methods in thermal physics, mechanics and analysis of the results of panoramic experiments, it is necessary to be a expert in physical optics; thermal physics and mechanics, as well as digital methods of processing and analysis of scientific images. In recent years, due to the continuously increasing volume of panoramic experiment visual data, the process of involving machine learning and artificial intelligence to analyze big data has begun. 
Images of flow fields in thermal physics are the main source of medium parameters information, configurations and dynamics of structures in a flow: vortexes, weak disturbances, strong discontinuities - shock waves, contact discontinuities, streamlines, turbulent structures, etc. The purpose of thermophysical flows visualization is qualitative and quantitative research, description of new phenomena and patterns in flows in various environments, as well as in their demonstration. The second important goal of accumulating visualization data of dynamic thermophysical flowfields is - to provide benchmarks for testing programs and algorithms for numerical modeling in thermal physics and mechanics.

Over the past 10-20 years, there has been a rapid transition to digital technologies for registration, processing, and analysis of dynamic processes in liquids, gases, plasma, multiphase media that arise in nature and technology. Relevant editions (reviews, papers, monographs) are published (and in the last 5 years they have been mainly posted on the Internet). Classic monographs and albums of flow visualization of the 80 - 2000s [3-8] are focused on the description of optical schemes, recording equipment, and analysis of test flow images. The visual information obtained in the 2oth century in the pre-digital period was mainly of a qualitative origin. In recent decades, the number of foreign review publications on the topic has increased many times (for example, [9-11] and others). Among the monographs and reviews of the last 20 years on panoramic methods in Russian, it should be noted [12-18].

In the works of the last decade, the focus is on methods of computer processing, tools, technologies for analyzing and recognizing of flow fields images, which make it possible to obtain quantitative information about flows. One of the goals of obtaining visual information about dynamic processes in flows is the creation of experimental data bases for verification of threedimensional calculation programs [19-20]. Actual problems of the development of an experimental base for verification of CFD codes when used in nuclear power [19].

With the advent of powerful computers, it became possible to carry out computational experiments based on the numerical solution of equations used in a mathematical model of the physical phenomenon or process under study. Scientific visualization systems allow presenting the calculations results and comparing them with experimental data [20-21].

The actual problem of analyzing the panoramic thermophysical experiment data is the problem of big data. A huge amount of digital information is accumulated in the experiments, obtained during video filming with digital cameras, thermal imagers, etc. When working with big data, the problem of machine learning is coupled when analyzing large data arrays (in our case, flow images). So far, very few papers have been devoted to this problem, but their number is growing rapidly.

Panoramic study of thermophysical fields includes a number of stages: 1. Visualization of the flow. 2. Registration of the image of the changing area (field) of the flow. 3. Digital image (film) processing. 4. Earning quantitative information. 5. Receiving the patterns of the physical parameter. 6. Analysis and interpretation, physical model.

At present, the development of methods for panoramic visualization and digital analysis of thermophysical fields is mostly determined by the introduction into experimental practice of modern software and electronic means for entering images obtained during visualization into a computer and their digital processing. With the help of specialized software, images are processed both in thermal physics and in fluid mechanics, medicine, geophysics, and biology. Similar basic tasks are posed: the noise level reducing on the original image, highlighting the structural elements of the objects under study, saving the results obtained in a form convenient for further work and presentation.

Especially a lot of quantitative information about the parameters of thermophysical fields has been obtained in recent years due to the use of cross-correlation image processing algorithms. These algorithms are the main in modifications of the Background Oriented Schlieren (BOS), Particle Image Velocimetry (PIV), including micro, stereo, tomographic modifications, seedless anemometry, thermographic PIV, when measuring velocity in viscous coatings, etc. 


\section{Methods, based on light refraction}

To visualize many types of optically transparent flows in thermal physics and dynamics of continuous media, visualization methods are used based on the phenomenon of light deflection when it passes through the density inhomogeneities of a transparent medium: the shadow method, schlieren method, interferometry, and their modifications. The optical refractive index of the medium $n$ is equal to the ratio of the speed of light in the medium to the speed of light in vacuum and is related to the local medium density by the Lorenz - Lorentz formula, which for gases is:

$$
\frac{n-1}{\rho}=k,
$$

where $\mathrm{k}$ is a constant value, (for air equal to $0.22635 \mathrm{~cm}^{3} / \mathrm{g}$ ).

The shadow method for detecting density inhomogeneities in a gas was proposed in 1867 by the German scientist Toepler. Schlieren visualization method is sometimes called the Toepler method [22].

If the gas flow is inhomogeneous, then the optical refractive index of the medium in the tested flow region depends on the coordinates $(x, y, z)$. When a flow region with a variable density is transparent, a ray propagating parallel to the $\mathrm{z}$ axis and passing through an inhomogeneity deviates from the initial propagation direction by an angle:

$$
\alpha \cong \int_{0}^{L} \frac{\partial}{\partial x} \ln n(x, y, z) d z
$$

The main disadvantage of shadow methods is that all density changes are summed along the propagation of the probe radiation beam direction, and thus the integral value of the density change is recorded. Therefore, shadow and interference methods are successfully used to visualize twodimensional, as well as some axisymmetric flows. When visualizing the gas flow field by the shadow method, the change in illumination is proportional to the degree of change in the gas density gradient. In the presence of strong density gradients in the flow (in particular, discontinuity surfaces), additional beam deflections occur on the discontinuity surface. The shadow image of the shock wave is a dark stripe on the side of the incident flow, which is followed by a bright light stripe, which intensity gradually decreases (Fig. 1). During laser probing, the appearance of diffraction fringes in the area of discontinuities in shadow images is possible.

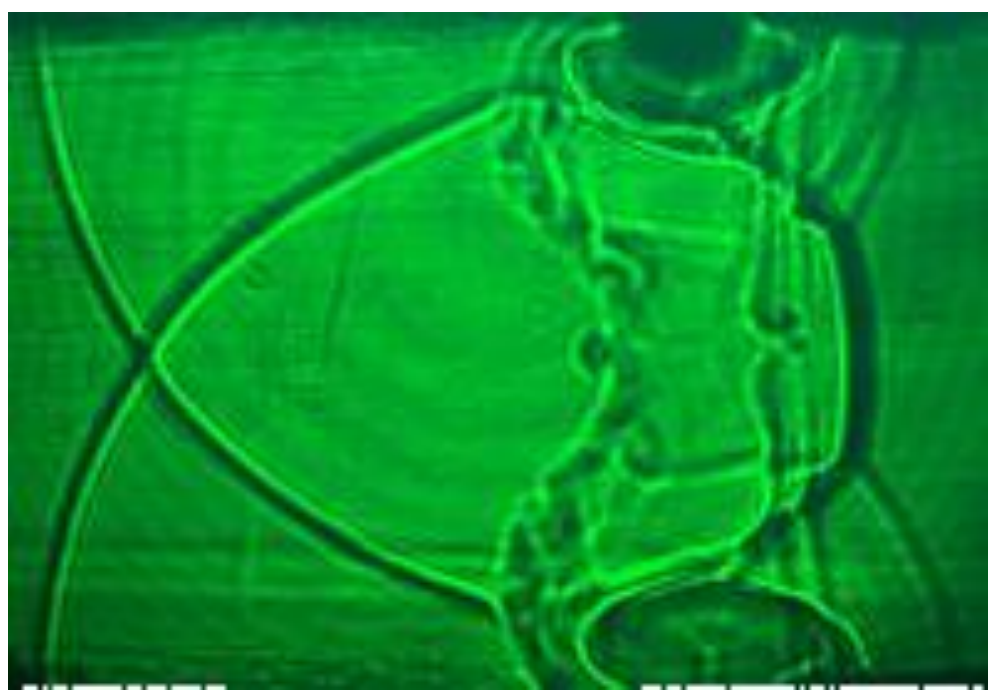

Fig. 1. Shadow image of a quasi-two-dimensional process of a shock wave interaction with a region of a pulsed surface discharge (laser illumination).

In [23], using the shadow method, four scenarios of circular hydrogen microjet diffusion combustion were investigated depending on the velocity of its outflow. In [24], a gas jet outflowing from a nozzle was investigated by shadow and schlieren methods. 
The schlieren visualization method (or Toepler's method) is a sophisticated shadow method. The basic principle of the schlieren system is that part of the light deflected when passing through the gas density inhomogeneity is retained by the edge of the knife installed in the focal plane of the beam that has passed through the region under study. On the screen, as a result, the illumination of the corresponding parts of the image will decrease or increase depending on where the deviation is directed. The change in illumination at a point associated with an inhomogeneity is determined by the value of the beam deflection angle, the focal length of the second lens, and the size of the light source. When visualizing the gas flow field by the schlieren method, the change in illumination is proportional to the gas density gradient in the studied area in the direction perpendicular to the edge of the knife, and not to the degree of density gradient change, as in the shadow method. Schlieren method visualizes better vortices, rarefaction waves; the position of the discontinuities is recorded more accurately by the shadow method.

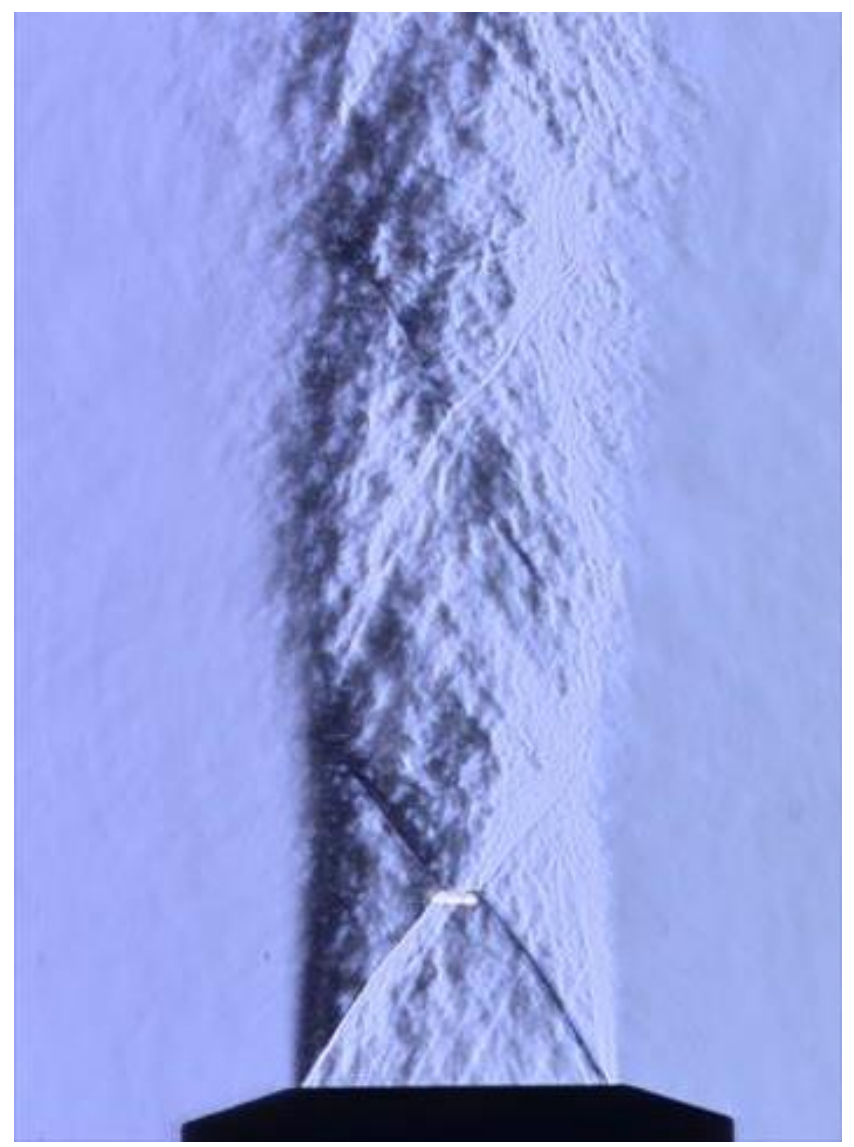

Fig. 2. Schlieren registration of a flow in a supersonic jet; optical discharge in a supersonic flow.

The visualization of a flow close to axisymmetric by the schlieren method can be considered using the example of an image of a gas jet (Fig. 2) outflowing from a nozzle [24].

Complicated modification of the schlieren method - the color schlieren method [25, 26]. There are several ways to get a colored shadow picture. Most often, a multicolor diaphragm is installed in the receiving part of the optical system or in the focal plane of the collimator. Colored schlieren images of the flow give more information about the flow field than black-and-white ones, since instead of one blackening density, three components change at once, namely, color, color saturation, and image brightness. The human eye is able to distinguish more color shades than gray gradations; therefore schlieren systems with a color image usually provide more quality information. The advantage of the color shadow method is that the details of the flow spectrum in the color picture can be easily distinguished from the boundaries of models and other objects that obscure the flow picture. 
Optical interferometry was used mainly for quantitative studies of the density of twodimensional transparent fluxes. With interferometric registration of the flow field, the distribution pattern of the light intensity bands reflects the distribution of the refractive index of the medium. The interference pattern is a system of bands, the distance between the maxima of which at a given wavelength is determined by the convergence angle of the interfering waves. The introduction of an optical inhomogeneity into one of the interferometer arms changes the optical path length of the corresponding beam in comparison with the unperturbed one and causes an interference fringes shift. Michelson, Zhamen, Mach-Zehnder, Fabry-Perot interferometers are used to measure density in liquid and gas flows. When studying two-dimensional flow regions (in which the gas parameters in the direction of rectilinear beam propagation do not change), the Fabry-Perot interferometer has increased sensitivity. When the interference fringes are shifted by $\delta$ (in fractions of the distance between the fringes), the density value changes by $\rho \Delta / \rho=\delta \lambda / \mathrm{kL}$, where $\mathrm{L}$ is the geometric length of the ray path in the inhomogeneity, the Gladstone-Dale k constant. The Mach Zehnder interferometer has some advantages in gas dynamics studies. In this interferometer, using a system of semitransparent plates and mirrors, an amplitude division of a light wave into two is carried out and the subsequent superposition of waves that have passed through different optical paths. For axisymmetric inhomogeneities, the displacement of the interference fringes in the cross section perpendicular to the flow symmetry axis is associated with a change in density by solving the Abel integral equation.

$$
\delta(r, x)=\frac{2 k}{\lambda} \int_{r}^{R(x)} \frac{\rho(y, x)-\rho_{0}}{\sqrt{y^{2}-r^{2}}} y d y
$$

where $\mathrm{R}$ is the boundary of the heterogeneity in the section; $\mathrm{r}, \mathrm{x}$ are cylindrical coordinates, $\rho_{\mathrm{o}}$ is the density of the unperturbed medium. A great difficulty in analyzing interferograms is to determine the change in density when crossing the discontinuity surface, especially for plane flows. It is difficult or impossible to establish the correspondence of the fringes in the interferograms when passing through the shock wave when shooting with a monochromatic light source. To solve this problem, there are special methods of interferometric measurements. Shadow visualization of the shock waves interactions with obstacles made it possible to establish the regularities of the restructuring of discontinuous flows. In particular, upon reflection of a shock wave from a solid surface, the existence of several types of configurations was found, which are realized under different conditions of reflection. For the first time this phenomenon was studied by E. Mach, research is being carried out now, since the question of the critical angles of transition from one type of reflection to another has not been solved unambiguously. It is important to analyze the density field in the vicinity of the point of intersection of the shock wave with the surface from the point of view of establishing the value of the critical angle. Therefore, investigations of the flow field of a flow upon reflection of a shock wave are carried out using both shadow and interference methods.
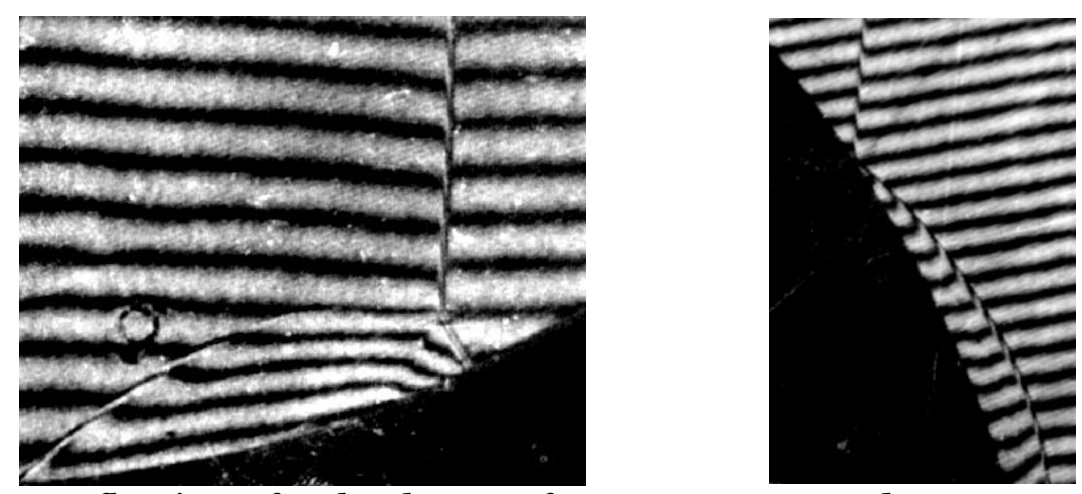

Fig. 3. Reflection of a shock wave from a concave and convex cylindrical surface. 
Figure 3 shows two interferograms (Gvozdeva L.G., and Sysoev N.N., 1970s) of a twodimensional unsteady flow - reflection of a shock wave from a concave convex cylindrical surface.

In [27], the results of interferometry of the inhomogeneity of the gas-dynamic windows of a laser with a supersonic flow of the active medium with the simultaneous registration of two interferograms with a mutually orthogonal shear direction are described. Data processing software has been tested.

Experimental time dependences of the radial distribution of electron density for a femtosecond laser microplasma of optical breakdown of gases (air, nitrogen, argon, and helium) at various pressures (from 1 to $10 \mathrm{~atm}$ ) were obtained using the probe microinterferometry technique [28].

Figure 4 shows an interferogram visualizing convective structures and a phase transition in a vertical layer of fresh water [29].

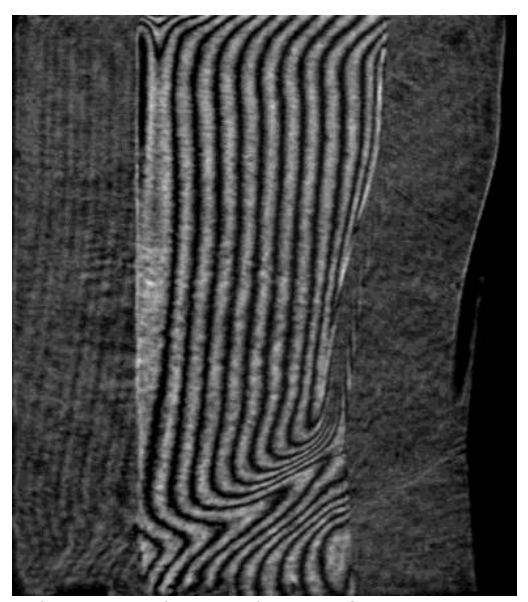

Fig. 4. Shear interferogram.

In [30], using a Mach-Zehnder interferometer, phase shifts were obtained from interferograms for a time of $1000 \mathrm{~ns}$ by the fast Fourier transform method, and then the refractive index values were derived using the Abel inversion. However, in general, the number of works on interferometry of thermophysical fields has noticeably decreased over the past decades. To some extent, the BOS method has come to replace interferometry.

Shadow and schlieren methods have been used since about the 19th century, but several important improvements have occurred in recent decades [31]. Shadow imaging is still the main panoramic imaging technique for ruptured and turbulent flows, including fluid flows. Figure 5 shows shadow photographs of typical events on the water surface, leading to the generation of drops [32].
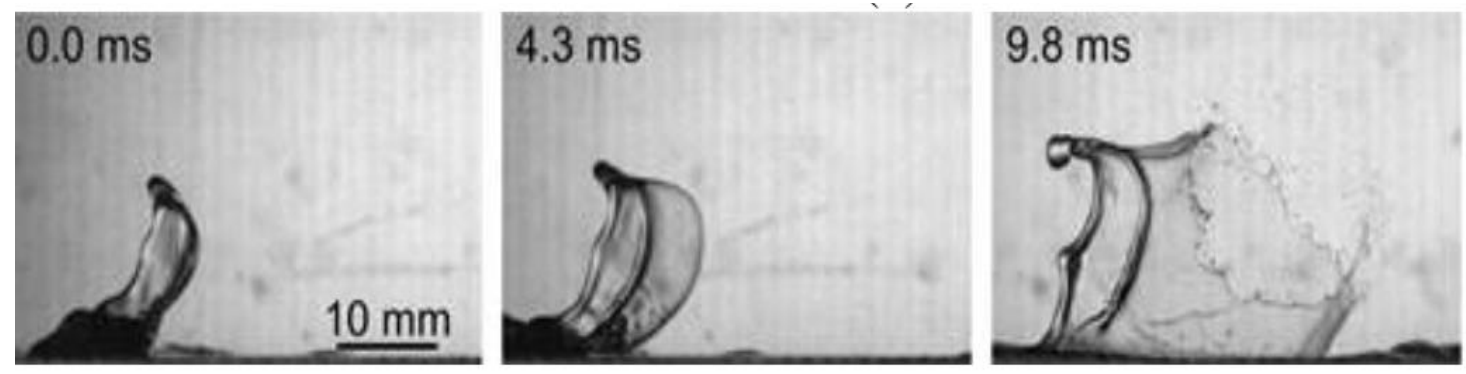

Fig. 5. Formation and rupture of structures during the droplets formation.

The use of high-speed digital cameras has made it possible to study long-term high-speed nonstationary processes with a shooting frequency of more than a million frames per second. Figure 6 shows 4 sequential shadow frames of the opposite motion of shock waves initiated by a surface discharge of nanosecond duration when shooting with a high-speed digital camera (124 thousand frames / s). 


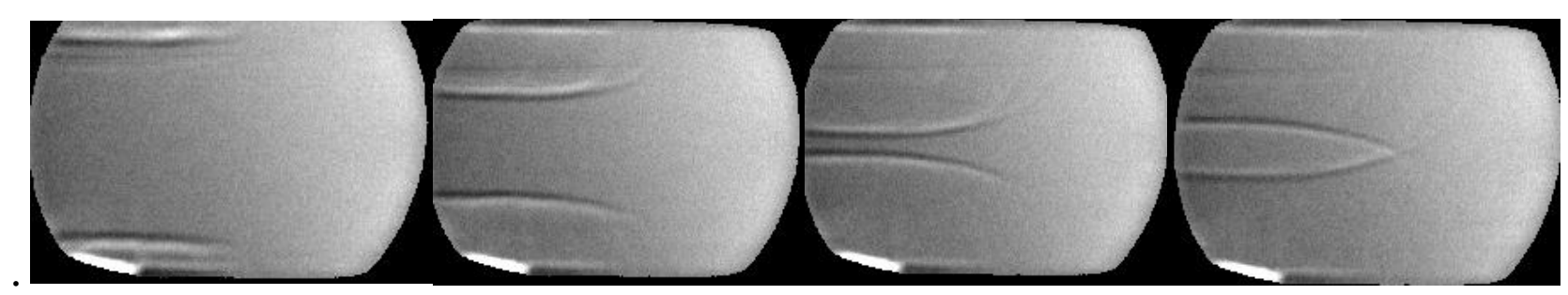

Fig. 6. Shadow recording of high-speed shooting of shock waves from a surface discharge.

\section{Gas discharge flow visualization}

The visualization of gas-dynamic flows by an electric discharge is based on the fact that the gaseous medium, being ionized, is a source of radiation (the phenomenon of electroluminescence), which intensity is related with local gas-dynamic parameters. When a stationary volumetric discharge burns in a gas flow, spatial density inhomogeneities cause a redistribution of the discharge plasma radiation intensity. This effect is often used to visualize supersonic flows in wind tunnels at low pressures [33]. The advantage of this method is the simplicity of the instrumentation and the possibility of observing dynamic changes in the flow pattern during the experiment. The flow density inhomogeneity leads to a redistribution of the electric current due to the strong dependence of the electron concentration and conductivity on the ionization coefficient value. The ionization coefficient is a non-linear function of the ratio of the electric field strength to the concentration of neutral particles E / N. Depending on the ratio of the linear scale value characterizing the particle concentration gradient to different characteristic physical scales (Debye radius, electron free path, etc.), various physical effects associated with gas density gradients are possible. Local radiation intensity of discharge plasma $\mathrm{I}(\mathrm{x}, \mathrm{y}, \mathrm{z})$ at a certain time instant is:

$$
I(x, y, z,)=\mathrm{f}(\mathrm{W}, \mathrm{U}, \mathrm{i}, \mathrm{N}, \mathrm{P}, \mathrm{T}, \mathrm{d}, \mathrm{Se})
$$

Where $\mathrm{W}$ is the electrical energy supplied to the discharge area, $\mathrm{U}$ is the voltage between the electrodes, $\mathrm{i}$ is the discharge current, $\mathrm{N}$ is the particle density, $\mathrm{P}$ is the pressure, $\mathrm{T}$ is the temperature, $\mathrm{d}$ is the distance between the electrodes in $\mathrm{mm}$, Se is the parameter characterizing the geometry of the electrodes. Since the 1950th, flow studies have been carried out around various models in TsAGI wind tunnels using a stationary high-voltage discharges [34-37]. In particular, stationary shock waves were visualized in the flow around the cone model on a cylindrical holder at different angles of attack. The visualized flow elements are vortexes in flow along the cone surface. Investigations of vortex flows were carried out around other isolated forms, form with a wing; a bow shock wave near a cylinder with a hemispherical head was visualized.

Since the 9os, in the works of Japanese researchers, the visualization of supersonic flows has also been carried out by the method of a stationary electric discharge. Shock-wave configurations were visualized around different models with a Mach number up to 10 [38-39]. A stationary discharge was realized between the linear and point electrodes. When the discharge passes through the section where the shock wave is visualized as a dark area. Cross-sectional images of the shock wave configuration relative to the model were obtained. To increase the area of the flow that can be visualized, additional point electrodes were introduced.

In India, work was carried out to visualize supersonic flows around models: (blunt cone, blunt cone with a spike and a disc [40-41]). The method of visualization by a stationary electric discharge was used, as in the previous case. The elements of the flow near the model were visualized: a bow shock wave, a point of attachment of a flow, a Mach stem. Numerical calculations were carried out for comparison with the obtained experimental data.

Nowadays, not many studies are carried out using the gas-dynamic flows visualization with the electric discharge method. Basically, visualization is carried out with a continuous electric discharge [42, 43]. Figure 7 shows an integral image of a supersonic rarefied nitrogen flow under illumination by a transverse glow discharge. At the shock front, during discharge burning in a supersonic stationary flow, layers with a space charge are formed, in which the electric field strength, the concentration of charged particles, and the gas conductivity change, and the nature of the change in the quantities depends significantly on the polarity of the field. 
It is known that discharge burning in a gas flow can lead to gas heating, significant changes in the structure and shape of discontinuities, an increase in the shock layer, an increase in weak disturbances, and a number of other effects, including those leading to qualitative changes in the flow structure. Thus, the visualization of the flow by a stationary gas discharge cannot be regarded as a contactless method.

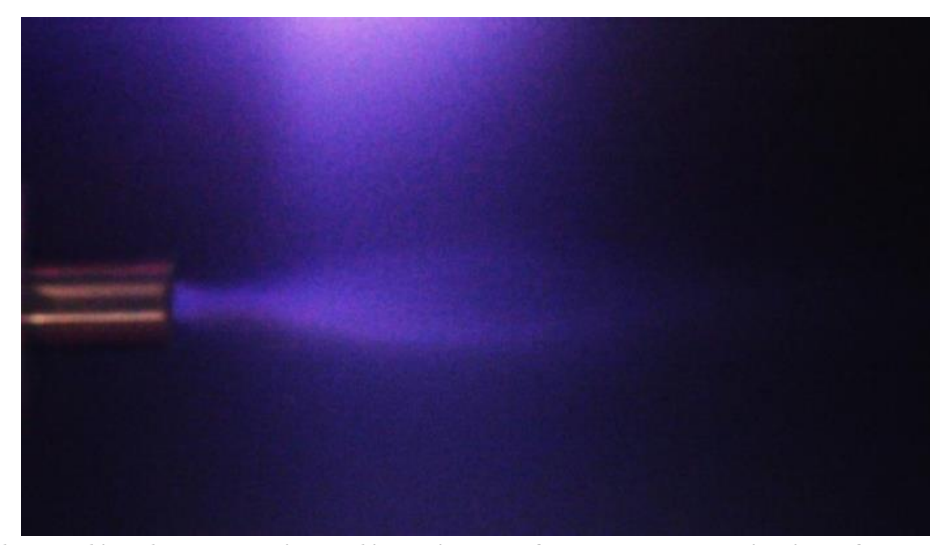

Fig. 7. Glow discharge visualization of a supersonic jet from a nozzle.

Some disadvantages of visualization by a gas discharge are eliminated by using a pulsed, initially spatially uniform discharge with a glow time much shorter than the characteristic times of gas-dynamic interactions. In studies on wind tunnels, these times are tens and hundreds of microseconds, in studies of fast gas-dynamic processes, including unsteady interactions of shock waves, in units of microseconds. When creating a nonequilibrium spatially uniform flow region, a pulsed volume discharge with preionization by ultraviolet radiation from plasma sheets is effective [44-47]. The use of such a discharge ensures the minimum time for the development of breakdown, the diffuse nature of the glow at the initial stages of the discharge development, and excludes the possibility of the inhomogeneities appearance in the discharge volume that can uncontrollably affect the gas-dynamic flow during image recording. The correct rectangular shape of the discharge region makes it possible to instantly ionize the section of the gas-dynamic flow, in particular, the flow in shock, wind tunnels. Figures (7-9) show the results of non-stationary and stationary gasdynamic flows visualization using such a discharge (MAI, Lomonosov Moscow State University, 2000-2020). Gas flows were visualized in a 48x24 mm shock tube channel with a specially designed test chamber of the same section. The two walls of the chamber are quartz windows; upper and lower - flat plasma electrodes $100 \mathrm{~mm}$ long, ignited at a given moment of the process; a volume discharge is simultaneously ignited. The glow time of the ionized air flow in the operating pressure range is 150 - $200 \mathrm{~ns}$. With integral registration of the flow field glow, the "exposure" is instantaneous from the point of view of the gas-dynamic time scale. During the exposure time highlighting the flow elements - at maximum shock wave velocities of $2000 \mathrm{~m} / \mathrm{s}$, the wave moves by $0.4 \mathrm{~mm}$, at average velocities of a plane shock wave of about $1000 \mathrm{~m} / \mathrm{s}$, the displacement is 0.2 $\mathrm{mm}$. Disturbances moving at a subsonic speed are "smeared" during the exposure by hundredths of a millimeter. Figure 8a shows an image of the flow field near a blunt cylinder $9 \mathrm{~mm}$ in diameter at the Mach number of supersonic flow $\mathrm{M}=1.5$ (artificial colors). Bow shock wave, oblique shock, reflected shock are visualized. In the photo $8 \mathrm{~b}$. the flow behind the passing shock wave (left) forms a separation zone at the sphere rear side. 


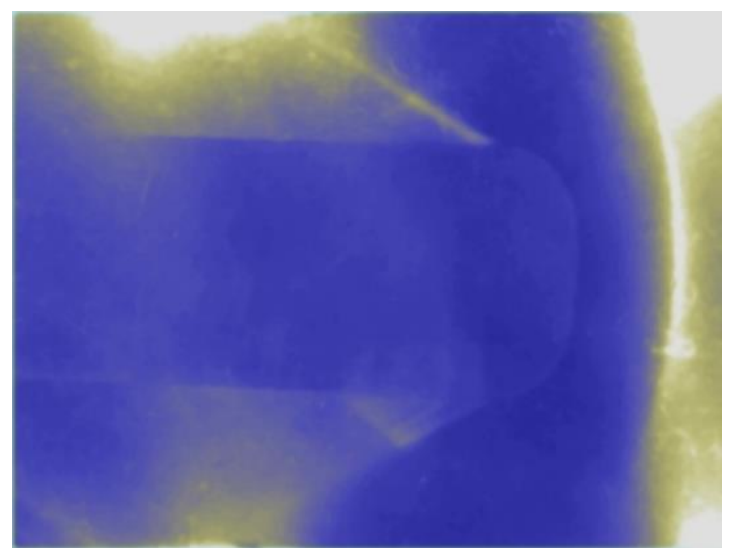

a

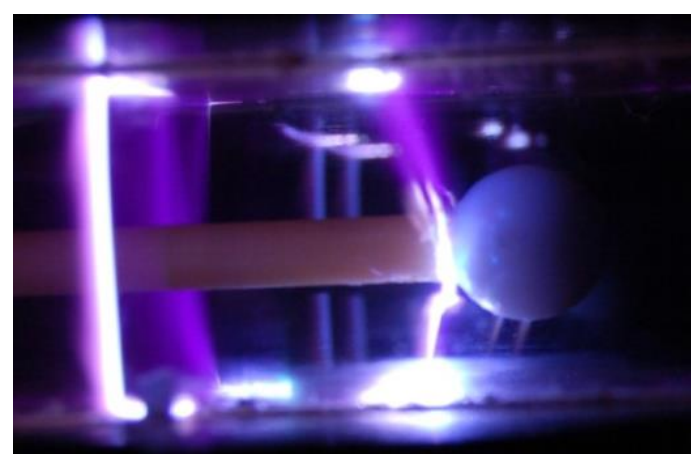

б

Fig. 8. A pulsed volumetric discharge visualization of a stationary flow near a blunt cylinder and an unsteady flow around a sphere.

Unlike optical methods, which require an object probing, this method allows visualizing the flow through one window of the test chamber. On the other hand, several images can be simultaneously obtained through both windows of the test chamber, while the total viewing angle can reach $200^{\circ}-250^{\circ}[44]$.

The visualization of irregularities using electroluminescence allows one to study threedimensional flow structures. Shadow methods can give an image of the flow lateral projection with the registration of integral characteristics in the transmission direction; the overlapping of the optical path by the model excludes probing of the central (axial) region. Fig. 9 shows two images of the flow around an axisymmetric model that combines the main geometric axisymmetric forms, which often become an object of research, being an element of many elements in aerodynamics. The model is a cone with a half angle of $10^{\circ}$, which is attached to the cylinder, then the diameter of the cylinder increases with a step. The photo in Fig. 9 shows an image of a supersonic flow near a model entirely placed in the discharge gap. The moment of the flow formation around the model after the passage of the shock wave with the Mach number $\mathrm{M}=2.8$ was recorded. The flow structure is completely visualized in two images recorded through opposite camera windows.
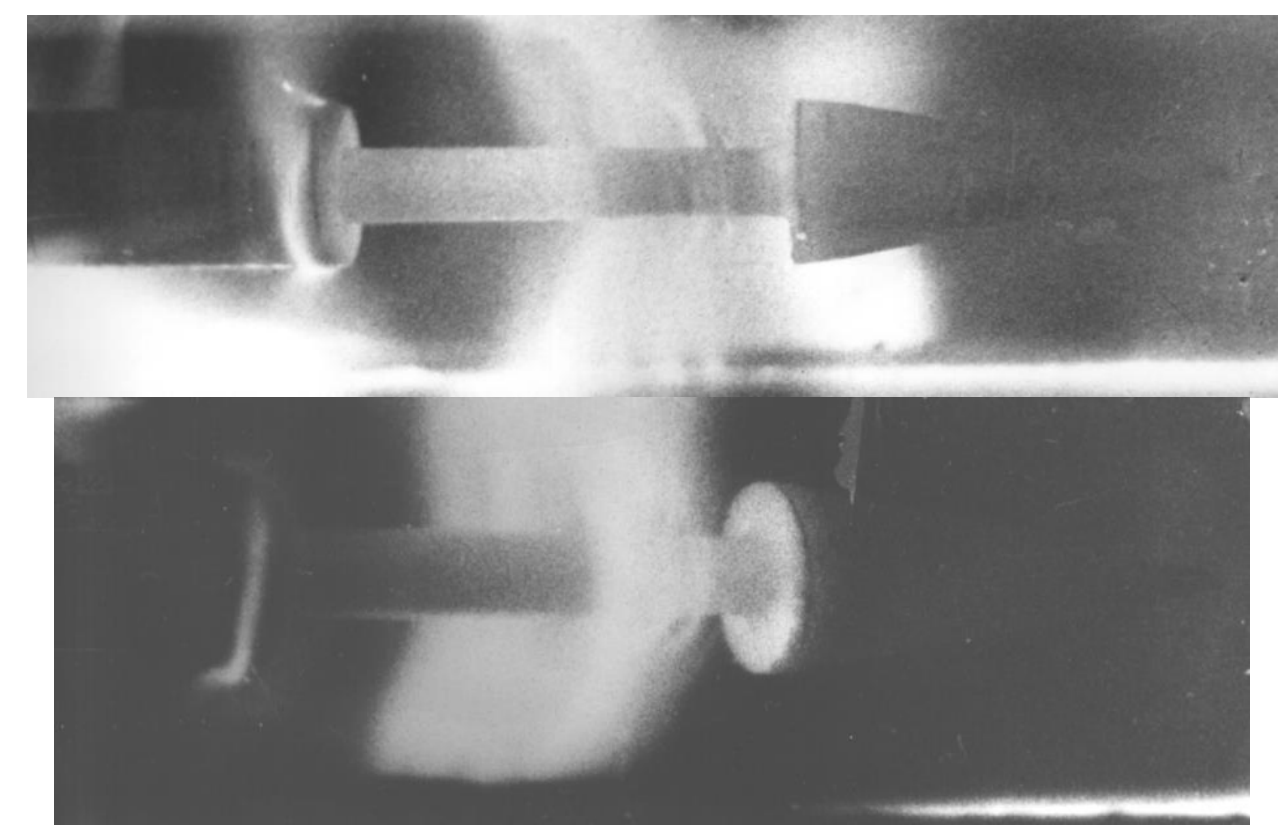

Fig. 9 Two-perspective visualization of the flow around the axisymmetric model.

The method of visualization by a pulsed volume discharge made it possible to study the dynamics of a three-dimensional vortex ring in the bottom region during diffraction of a shock 
wave on a cone with a step [45]. We also visualized unsteady quasi-two-dimensional flows in a channel with steps, which arises in the channel behind the incident shock wave. On the upper and lower walls of the channel from glass to glass, there were rectangular obstacles $2 \times 6 \times 48 \mathrm{~mm}$ [46, 47]. In Figure 10, a pulsed volumetric discharge visualizes a plane wave bending around an obstacle on the bottom wall and a cylindrical vortex in the separation zone. The volume discharge glow is instantly redistributed to the low-pressure zone in front of the shock wave and to the separation zone behind the step.

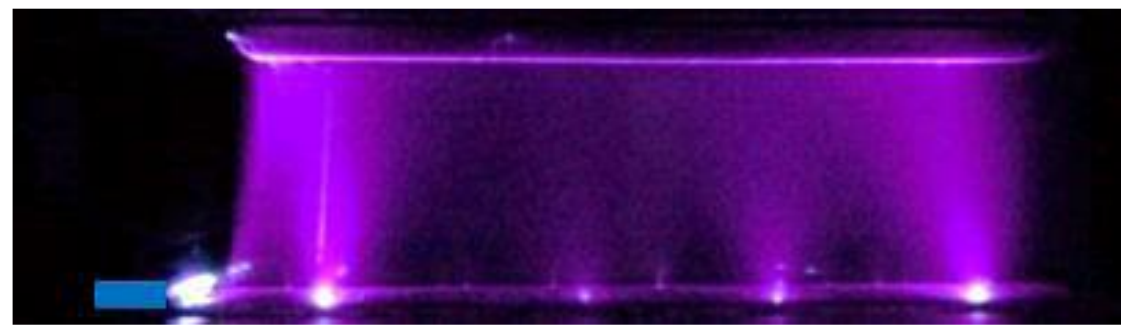

Fig. 10. Flow of a pulsed volumetric discharge with preionization during diffraction of a shock wave by an obstacle on the lower wall.

\section{The problem of big data in the analytic data of panoramic visual information}

The actual problem of a panoramic experiment data analysis is the problem of big data. In the experiments today a huge amount of digital information is accumulated, obtained during video filming with digital cameras, thermal imagers, etc. The development of digital technologies leads to a multiple increase in the data array on the parameters of thermophysical flow fields; large arrays of digital data appear which make it impossible to process it manually. Thus, modern video films recording the evolution of turbulent fluid flows based on shadow methods, tracing, thermography require processing and qualified analysis. The transition to another level of data analysis is inevitable.

There is a need to automate the process and data analysis - experimental images of thermophysical fields, using various approaches, including machine vision and learning methods using deep learning, convolutional neural networks (CNN). So far, there are few works devoted to this problem, but their number is growing rapidly. Work in this direction begins in a number of scientific laboratories. Software is being developed to recognize the structural elements of flows in gases, liquids and plasma. For software testing and training of neural networks, arrays of images of various currents are used, registered by shadow, schlieren, and PIV methods.

Today, the most promising approaches to solving these problems are based on digital image processing with various algorithms for edge detection and object recognition [48] to identify complex stream structures. Various image edge detection algorithms are suitable for shock wave detection. Various researchers have shown that the Canny algorithm [51] is best suited for processing shadow and schlieren images. The authors of [48] developed software for detecting and tracking shock waves. The mixing process in the nozzle improved by means of boundary detection in schlieren images was analyzed in [52]. Image processing was performed using the Canny algorithm. Work is underway to improve the quality of images by removing noise, subtracting background images by various methods. It was shown in [53] that subtraction of the background image in frequency representation (performed using the fast Fourier transform) yields the best quality. Also promising for digital processing of experimental images by computer vision methods are methods of image segmentation using algorithms such as k-means, energy minimization, etc .; feature detection methods (SURF, LESS, HOG, etc.).

Currently, the topic of machine learning for fluid dynamics is actively developing. A fairly detailed review of research on this topic is given in [54]. An approach based on machine learning for identifying flow structures in schlieren images is also developing. In [55], a system for classification and processing of schlieren images of objects in a wind tunnel is proposed. The system 
was able to extract three parameters from the images: the angle of refraction of the bow shock wave, the difference in line intensity and the average line width. Based on these data, the system calculates the flow velocity near the model. Neural networks can extract not only shock waves, but also any other elements. For example, the authors of [56] have successfully applied a neural network to classify wake vortexes behind a wing profile. Neural networks are beginning to be used to predict [57] or reconstruct [58] flow dynamics. New physics-based methods for calculating the loss function [58]. Deep machine learning allows simulation of turbulence and other largedimensional gas-dynamic systems [59].

To study the evolution of gas-dynamic flow over 6-10 milliseconds, in [60-61] results of quantitative analysis of high-speed shadow shooting shock tube flow were tested. Using machine vision and learning [61] [62], three programs for processing shadow and schlieren images were developed. The first one works on machine vision algorithms, the second uses the convolutional neural network; we trained to recognize and automatically track various flow structures, the third program uses the cross-correlation method to estimate the flow velocity from the displacement of turbulent structures and particles suspended in the flow - by analogy with the PIV method. For edge detection, the Canny edge detection algorithm was used, which turned out to be the most effective for analyzed shadow and schlieren images. The algorithm for finding the angle of an oblique shock wave includes the selection of boundaries, the search for equations of straight lines (shock waves) using the Hough transform, and filtration of the found straight lines by length, angle, and position. The moment of transition to the subsonic regime has been determined. The dynamics of plane shock waves was automatically measured using a convolutional neural network. The speed of objects recognition in images (shock waves, tracer particles in a flow) by the neural network was $15 \mathrm{fps}$. The time dependences of the incident speed, reflected shock wave were automatically measured and plotted. The dynamics of a pseudoshock train in a channel is investigated. The use of machine vision and learning algorithms made it possible to speed up the processing and analysis of large arrays of experimental digital images (Fig. 11) and to fully automate this process. The manual processing of one shot of the flow in the shock tube (about 1000 frames at a shooting speed of 150,000 frames / s) could take a whole working day. The developed software solves this problem in one or two minutes. Thus, the acquisition of new physical information was significantly accelerated.
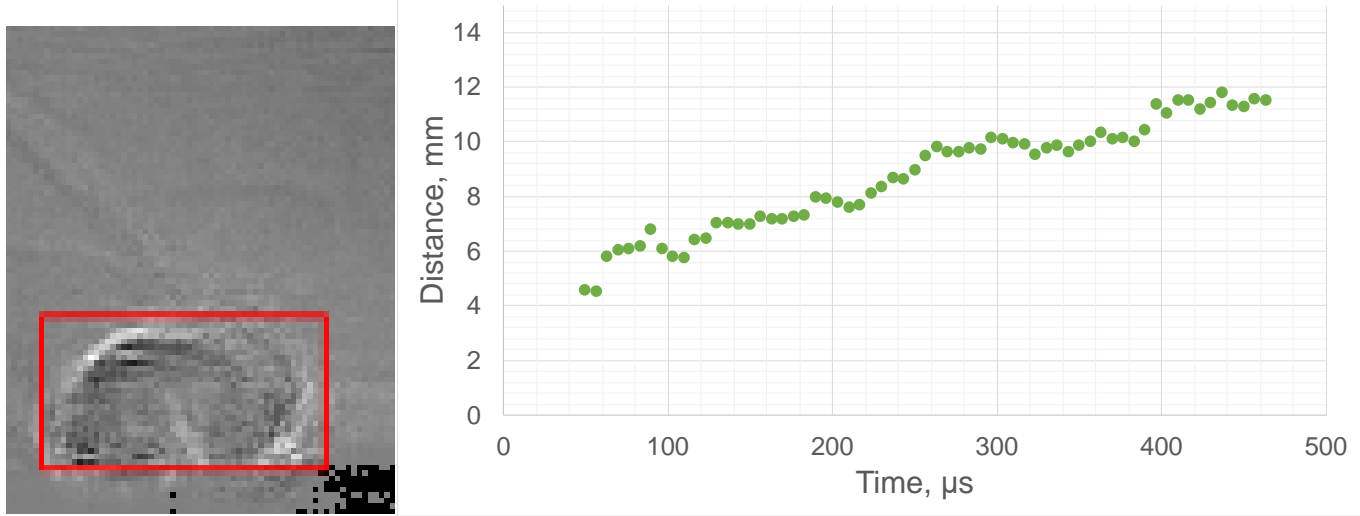

Fig. 11. Recognition of a plume from a pulsed surface discharge using CNN and an automatically plotted graph of its growth.

\section{Application of infrared thermography}

The method of infrared (IR) thermography of thermal fields is based on measuring the distribution of thermal radiation and converting it into a temperature map. Thermal radiation arises in solids, liquids and gases at temperatures above absolute zero due to vibrations of atoms or rotational-vibrational motion of molecules [63]. As it is known, objects can absorb, reflect or transmit radiation energy. Corresponding coefficients are introduced to describe these physical processes. IR radiation is in the range of the electromagnetic waves spectrum from $0.75 \mu \mathrm{m}$ to 1000 
$\mu \mathrm{m}$ between visible light and radio waves. In turn, the IR spectrum is usually subdivided into shortwavelength $\left(0.75^{-1.5} \mu \mathrm{m}\right)$, medium-wavelength $\left(1.5^{-20} \mu \mathrm{m}\right)$, and long-wavelength regions (20$1000 \mu \mathrm{m}$ ), although in the literature there are different divisions of the IR range depending on the further application.

Infrared thermography is a non-contact method for measuring and analyzing the thermal radiation of objects or flows. Registration of thermal radiation provides extensive information about the energy state of the object of study, which is used in thermal physics, medicine, geology, biology, energy saving, etc. This method is used in such engineering applications as regulation of thermal insulation, non-destructive testing, etc. Fig. 12 shows examples of thermograms: heat outflow through the slots of the window frame and the image of an "energy-saving" lamp; thermal imager was with a registration range of $3.7-4.8 \mu \mathrm{m}$.

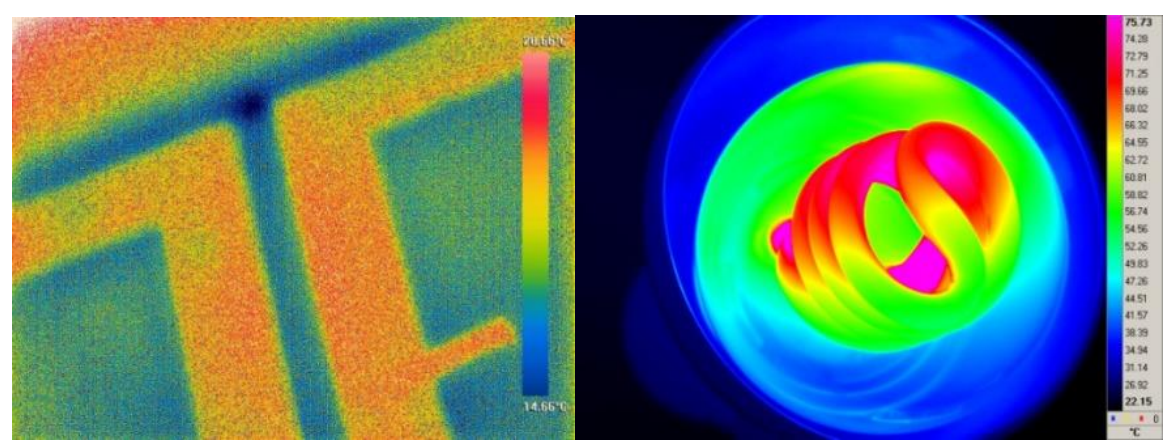

Fig. 12. Thermograms of the thermal fields: window frame and an energy-saving lamp.

Thermography is used to diagnose the human psychoemotional state in the infrared (and terahertz) range [64 - 66].

The increased interest in thermography is due to both the emergence of new generation of thermal imagers and the possibilities of digital processing, analysis, and storage of thermographic images and films. The total flux of thermal radiation $\mathrm{W}$ recorded by the thermal imager is equal to:

$$
\mathrm{W}=\tau_{\mathrm{a}} \varepsilon_{\mathrm{o}} \mathrm{W}_{\text {Black body }}\left(\mathrm{T}_{\mathrm{o}}\right)+\tau_{\mathrm{a}}\left(1-\varepsilon_{\mathrm{o}}\right) \mathrm{W}_{\text {Black body }}\left(\mathrm{T}_{\mathrm{f}}\right)+\left(1-\tau_{\mathrm{a}}\right) \mathrm{W}_{\text {Black body }}\left(\mathrm{T}_{\mathrm{a}}\right) \text {. }
$$

This is a common measurement formula used in most commercial thermal imaging systems. The thermal imager gives an uncorrected value of the object temperature, obtained taking into account all the thermal radiation received by the detector. Thermography can be used to heat transfer experimentally study on flat and relief surfaces with different geometries [67, 68].

Despite the fact that gaseous media are transparent in the IR region of the spectrum, thermography is widely used to study air flows and their effects on surfaces. The earliest known attempts to measure heat transfer coefficients in a high-speed air flow using thermography were carried out in a hypersonic mode in a wind tunnel [69]. In [70] implemented shadow visualization of rarefied flows in infrared light (gas density in the shock region $\approx 10^{-3} \mathrm{~kg} / \mathrm{m}^{3}$ ), which is not available for visualization in visible light. Since molecular nitrogen cannot emit in the infrared range, the visualization of shock waves $(M=21)$ is associated with the refraction of infrared radiation reflected from the rear wall of the test chamber at the shock waves. There is a "direct shadow" visualization of the flow in the infrared range, where the refractive index of gases increases significantly.

One of the important applications of thermography is the determination of the area of a laminar-turbulent transition on a surface in a gas or liquid flow. The problem of controlling the laminar-turbulent transition in gaseous media is of great interest for optimizing the geometry of aircraft, both from the point of view of gas dynamics and from the point of view of heat transfer on a surface in a streamlined flow. Thermography made it possible to determine the zones of laminarturbulent transition in flows around aircraft by measuring the temperature maps on the wings and blades [71-73].

The work of a group from Dryden Flight Research Center described a flight experiment to study the pressure distribution when flowing around a flat plate in flight at supersonic speeds up to Mach 2.0 [74]. The boundary layer was investigated in flight. The aim was to determine the 
characteristics of the boundary layer transition and the efficiency of the surface coating for future flight tests using IR thermography. Infrared imaging was used to record the shock wave incident on the surface in addition to determining the transition line of the laminar boundary layer to the turbulent one.

An important area of thermography is the analysis of near-surface fluid flows. In the literature, there are mainly papers devoted to the study of the gas-liquid interface configurations and slow flows with low Reynolds numbers. IR radiation is absorbed directly on the surface of the liquid. Thermography can be used to measure the temperature fields of a liquid in laminar and turbulent modes of convective flows from the free surface of a liquid [75]. The space-time characteristics of multiscale convective structures were obtained [76].

The modes of air flow and liquid flows in inclined pipes were investigated using infrared thermography on the basis of thermal images and fields of local heat transfer coefficients on a heated surface [77]. In [78], IR thermography, together with the PIV method, is used to analyze the structure of a free liquid jet falling on a metal plate in air.

A large number of works are devoted to the use of infrared thermography for studying the heat transfer of jet flows by registering the heat flux from the outer surface of the wall [79]. Of practical interest were the problems of monitoring and scanning the temperature field of the outer mixers walls during the flow of water and liquid metal coolants [77-81]. For impact jets, measurements of the spatial and temporal characteristics of a turbulent water flow were usually carried out through a thin metal wall (plate, foil) [82-85]. The averaged thermal fields obtained due to heat transfer of the investigated flow to a solid wall were recorded. The problem with measurements through metal substrates is the attenuation of temperature fluctuations by the test surface. In [86], it was proposed to compensate for the attenuation of temperature pulsations by restoring the initial heat signal from the inner side of the surface by solving the inverse heat conduction equation.

The development of high-speed thermographic technology has led to the possibility of registering sufficiently fast processes, in particular, the characteristics of heat transfer in a turbulent flow. In [87], IR imaging is used to visualize the turbulent flow of water in acrylic round pipes with a high temporal resolution. In [88], the starting processes and dynamics of a nonsubmerged high-speed liquid jet on a waterjet cutting machine were recorded. The research was aimed at obtaining new data on two-phase flows in extreme conditions, and can be applied to improve engineering hydraulic jet structures. Figure 13 shows two thermographic images of the development of a supersonic hydro jet when shooting with a frame rate of up to $415 \mathrm{~Hz}$; observation is carried out from the peripheral jet region and its air-water covering. The outflow velocity of the jet on the axis reaches $270 \mathrm{~m} / \mathrm{s},\left(\operatorname{Re} \approx 10^{7}\right)$.
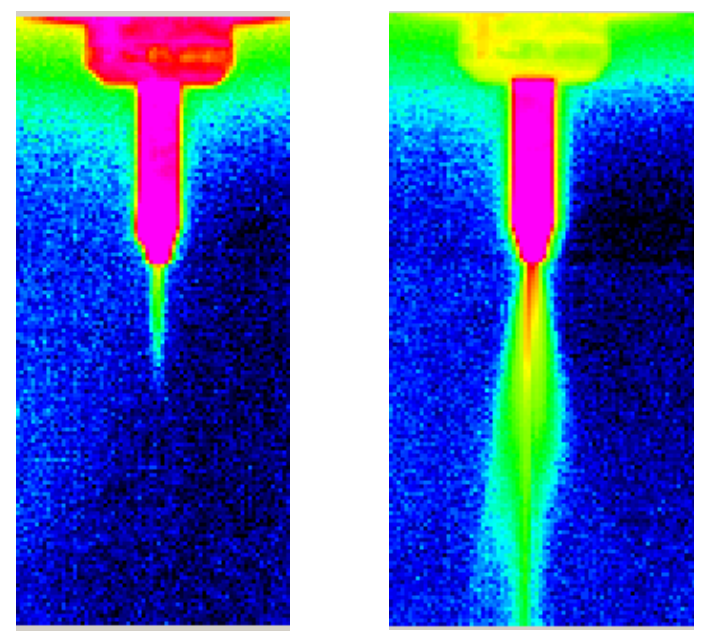

Fig. 13. Thermograms of high-speed hydraulic jet starting processes in the period up to $0.005 \mathrm{~s}$ after starting.

The property of water to absorb infrared (IR) radiation on a submillimeter scale has made it possible to propose a method for studying non-isothermal unsteady turbulent fluid flows in a 
boundary layer [89] based on IR thermography. When registering through an IR-transparent window, the method allows visualizing thermal radiation from a thin near-surface layer of a liquid. It is shown that through a wall transparent to infrared radiation during a thermal imaging study of a moving liquid, the pulsating, energy characteristics of a non-isothermal turbulent boundary liquid layer can be measured with a frequency resolution of $100 \mathrm{~Hz}$. For the model of a flat Tjunction, the presence of inertial intervals of energy spectra in the range from 4 to $40 \mathrm{~Hz}$ was revealed. The application of the method for studying impact jets is described in [90-91]

Figure 14 shows an example of the transition region visualization of an impact jet flow. Time sweeps of temperature pulsations at four different distances from the stagnation point are shown.
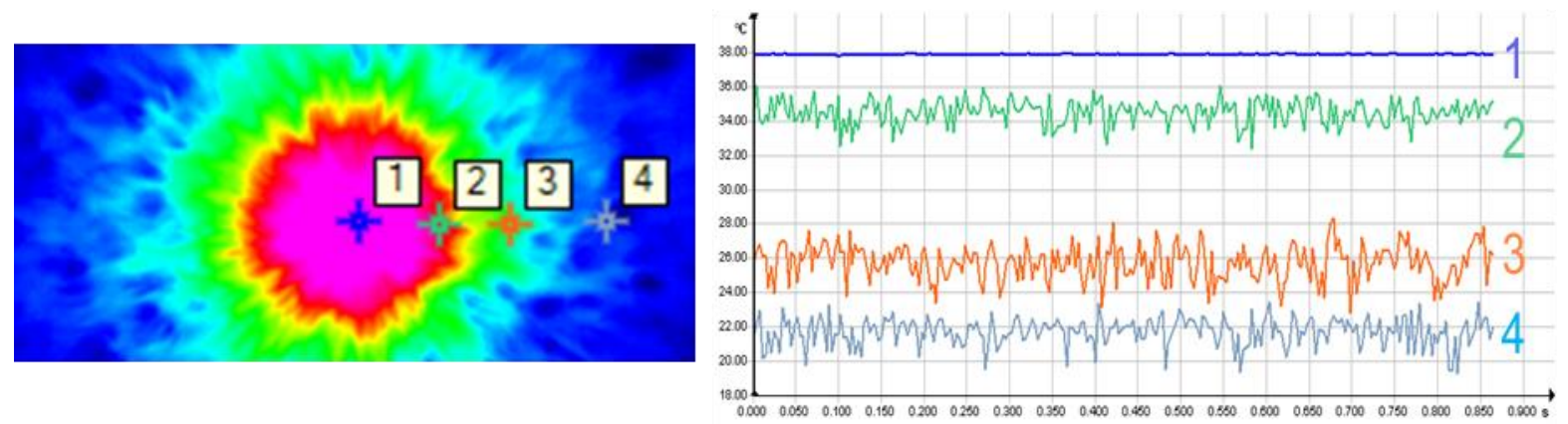

Fig. 14. An example of an instantaneous thermogram and temperature evolution at four different distances from the stagnation point.

\section{Background oriented schlieren method}

The Background Oriented Schlieren method which is based on the physical phenomenon of refraction and cross-correlation analysis of images deserves a separate consideration. When visualizing the fields of the refractive index of transparent inhomogeneous media, the displacements of the background points separated in the image space, placed behind the object under study, are determined. Quantitative measurements of density fields are possible in the case of a two-dimensional and axisymmetric flow of a transparent medium using the photometric shadow method. The angles of light deflection are related to the distribution of the refractive index within the studied inhomogeneity by means of the Abel relations. For example, in Ref. [92], the density fields of helium were measured for a hypersonic flow around a cone.

In English-language literature, method is known as BOS (Background Oriented Schlieren). The method was proposed almost simultaneously by Mayer [93] and Daltsil [94], at the same time the first experimental surveys were made. The predecessor of this method can be considered the technique of speckle photography, which was developed at the Institute of Heat and Mass Transfer (ITMO) named after V.I. A. V. Lykov of the Academy of Sciences of the BSSR since the mid-1980s. Quantitative visualization of flows based on speckle technologies is described in [95]. Techniques for digital speckle photography have also been developed in the PIV technique, Talbot interferometry, [96].

The essence of the BOS consists in comparing two images of the same background, taken in the absence and in the presence the investigated transparent object with inhomogeneities between the camera and the background of $[97 ; 98]$. The background screen must meet certain requirements in order to obtain high-quality data with the maximum amount of useful information and a low noise level during further digital processing of experimental images.

A change in the refractive index along the observation line in the case of shooting the background through an object leads to a discrepancy between the reference and "working" images (Fig. 15). By analyzing the displacement of the background elements in the images, one can obtain quantitative information about the integral change in the refractive index of the medium under study along the observation direction. Cross-correlation methods for digital comparison of experimental images have previously been significantly developed within the framework of the digital tracer anemometry (PIV) method. 


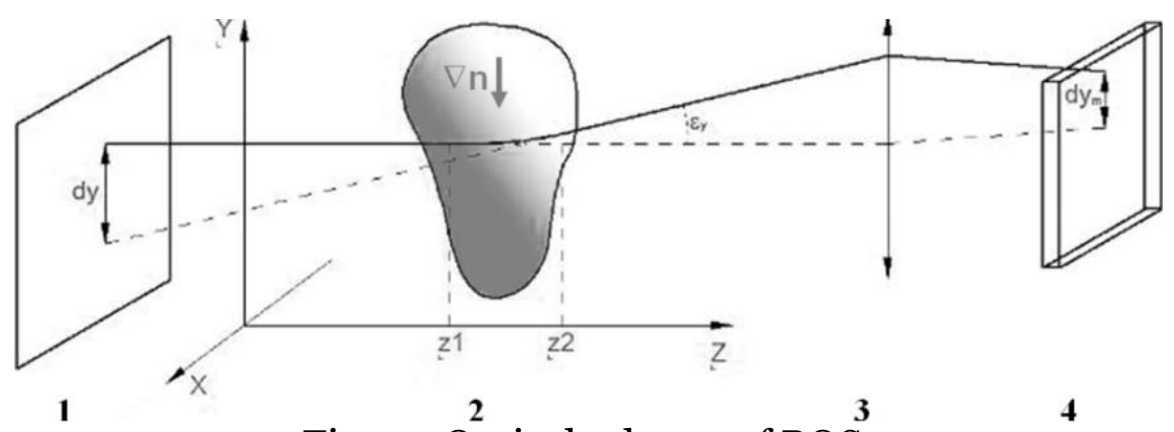

Fig. 15. Optical scheme of BOS:

1 - background, 2 - object under study, 3 - lens / objective, 4 - digital recorder

The Y-component of the beam deflection coming from the background is expressed from the law of refraction as follows:

$$
\varepsilon_{y}=\int_{z 1}^{z 2} \frac{1}{n} \frac{\partial n}{\partial y} d z \approx \frac{1}{n_{2}}\left\langle\frac{\partial n}{\partial y}\right\rangle\left(z_{1}-z_{2}\right)
$$

Then the corresponding background element will be displaced in the working image relative to the reference one by the amount:

$$
d y=\operatorname{tg} \varepsilon_{y} \cdot L_{b} \approx \varepsilon_{y} L_{b}
$$

The relationship between the density of a homogeneous gas and its refractive index can be expressed by the Gladstone-Dale relation:

$$
\frac{n-1}{\rho}=G
$$

where $\mathrm{G}$ is the Gladstone-Dale constant. The recorded BOS displacement of the background element in the image is directly proportional to the density gradient in the plane perpendicular to the optical axis.

$$
d y \approx\left\langle\frac{\partial \rho}{\partial y}\right\rangle \frac{G}{\langle n\rangle} l_{z} L_{b}
$$

Here $L_{b}$ is the distance from the object to the background, $l_{z}=\left|z_{1}-z_{2}\right|$ is the thickness of the flow under study along the scheme optical axis. The displacement of the image is influenced by all flow elements along the optical beam - in fact, information about the studied flow is averaged along it. Reconstruction of the density field directly from the results of one-angle BOS is possible only for a flow close to two-dimensional (which is rare in the experiment), or for an axisymmetric flow (based on the use of the inverse Radon transform.).

To obtain quantitative values of the density field in complex three-dimensional flows, a multiangle BOS survey is required with subsequent reconstruction of the three-dimensional field from two-dimensional images using tomographic reconstruction approaches. In this case, the shooting is carried out simultaneously from several (usually at least 5-6) angles. For stationary flows, the survey can be performed sequentially from different angles with the same camera [99]. However, even the displacement fields obtained on the basis of BOS (associated with density fields) can serve as a useful source of information about thermophysical objects, for example, data on the position and dynamics of characteristic gas-dynamic structures (discontinuities, vortices, etc.).

In Russia, the first work with the use of BOS was carried out in the early 2000s at the Institute "MPEI". Unlike shadow methods, BOS visualization does not require the use of optical elements comparable in size to the object under study. This makes it convenient for field research and experiments, and other cases when visualization of large-scale currents is required. The authors of [100] used BOS together with PIV for combined visualization of a flow with a Mach number M $=8$ in a wind tunnel. BOS studies of unsteady transonic flows containing shock waves in the channel 
and at the exit from the shock tube channel were carried out at Lomonosov Moscow State University $([101,102]$. Figure 16 shows 3 BOS images of the shock wave exit and the flow from the shock tube end. - distance up to $40 \mathrm{~cm}$.
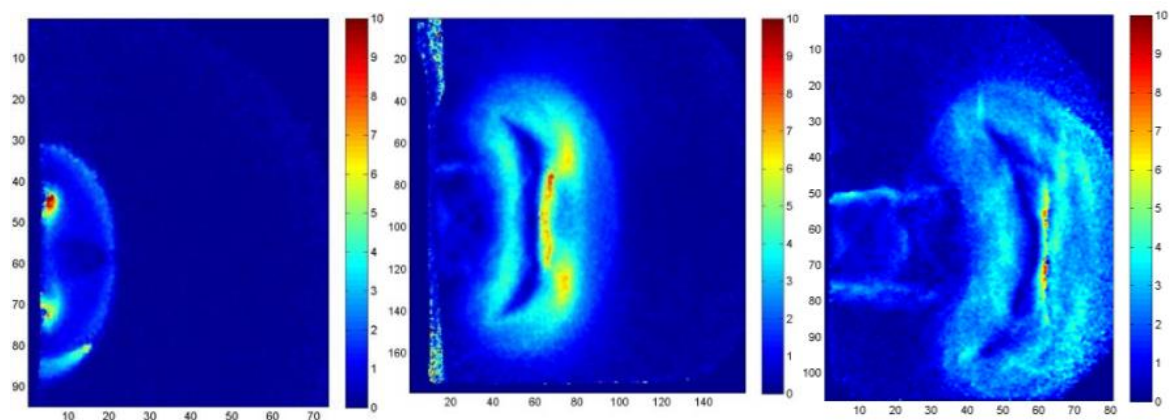

Fig. 16. BOS images of a supersonic flow at the exit of a shock wave into the atmosphere.

In the study of combustion processes, BOS can also be widely used, since the method is able to visualize not only heat fluxes and flames, but also to determine the concentrations of components in various fuel mixtures [103]. BOS was also used for full-scale field survey of explosive tests, [104 - 106]. The main result was the determination of the shock front generated by the explosion dynamics. The results of the work unambiguously indicate that the BOS has exceptional capabilities for practical field applications, although it is not always possible to make quantitative measurements.

Almost from the very beginning of the use of the Background Oriented Schlieren, significant errors were found that arise when visualizing strong density gradients (in particular, shock waves) by this method. The results of numerous studies show that the quantitative determination of the density jump at the shock front using the classical BOS scheme is a difficult problem. The effect recorded at the shock fronts often does not correlate with the physical parameters of the flow. In papers [107], [101] it was shown that this problem is actually caused by the fact that the detected value of refraction goes beyond the sensitivity of the method. Due to the strong light refraction at the shock front, the deflected beam can go beyond the optical scheme and not be detected.

To date, most of the work related to BOS considers flows and processes occurring in gas. However, by its principles, BOS is also applicable to the study of processes in transparent liquid and amorphous transparent media. Thus, the method was used to register internal waves in water [108] and thermal processes in water and plexiglass (MPEI 2008). Fig. 17 shows the images of the displacement fields during the movement of convective thermals in a flat vessel.
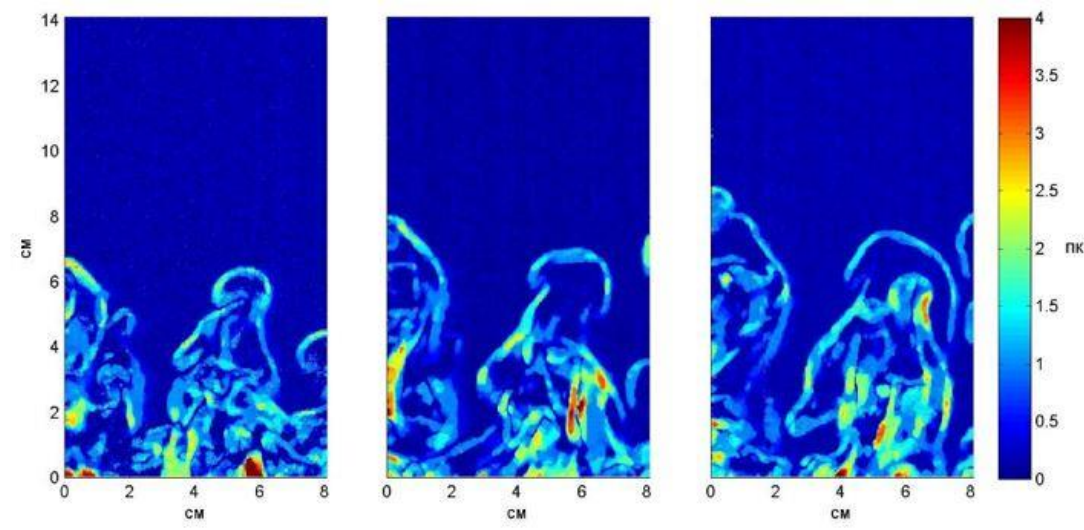

Fig. 17. BOS images of convective flows in water.

BOS occupies the position of classical qualitative shadow methods, outperforming them due to the greater ease of use [109] where high spatial resolution is not required. The greater simplicity of the BOS hardware in comparison with other methods makes multi-angle BOS visualization more accessible. 
With all the advantages of BOS, a number of drawbacks are obvious [110, 111]. Algorithms for cross-correlation of images are averaged over the size of the polling window; TMF always provides a lower image resolution than comparable traditional shadow schemes. Increasing the sensitivity requires a larger interrogation window and thus resolution decreases. Resolution loss is most noticeable for objects such as shock waves and interfaces; while traditional shadow methods produce a finished image in real time (no processing), BOS requires processing. Problems with focusing simultaneously on the object and background arise. Method is also sensitive to vibrations. One of the drawbacks complicating quantitative measurements using the BOS is the nonparallelism of the light beams probing the volume under investigation, which leads to the nonuniformity of the spatial scale of the BOS fields depending on the position of the fixed irregularities along the optical beam. The disadvantage of introducing a large converging lens into the optical system, matched with the lens of the recording camera, cancels one of the essential advantages of the method, since it limits the dimensions of the investigated inhomogeneity to the dimensions of the main optical element.

\section{PIV}

The movement of gas and liquid elements can be visualized by introducing marked, colored particles, plumes of smoke, threads, silk threads, etc. into the flow. etc. This visualization method - the tracing method - is one of the oldest methods of direct flow visualization. With the integral registration of a particle trace in a gas, the tracing method allows one to observe the trajectories of particles, streamlines. By registering with the exposure $\delta t$ the movement of tracer particles in the flow, it is possible to obtain images of the segments of the path $\delta$ s traversed by the particles in $\delta$. The quantity $\mathrm{v}=\delta \mathrm{s} / \delta \mathrm{t}$ is the average velocity of the particle in this segment. Digital processing of tracing results has developed into a special direction providing the reconstruction of the dynamics of three-dimensional velocity fields - the PIV method (particle image velocimetry).

The method of digital tracing (anemometry from particle images, Particle Image Velocimetry method) is based on statistical analysis of particles moving with the flow displacements of images under study, visualizing the flow over a short time interval when registering these particles in a selected plane using an optical knife. The optical knife (laser radiation) is formed in the form of two short pulses with a certain time interval between radiation pulses. The result of PIV method measuring is the instantaneous flow velocity fields. Many reviews and monographs are devoted to the description of modifications and method applications [112, 114]. In Russia, the method has been actively developed since the 90 a the Institute of Thermophysics named after V.I. S.S. Kutateladze RAS. The review [15] provides an analysis of the history and current trends in the development of the PIV method in applications to aerodynamic installations. The authors consider the basics of the anemometry method, options for its implementation, history and current state of hardware.

The appearance of the term PIV dates back to the 8os, when it was identified as a special case of the LSV (Laser Speckle Velocimetry) method [115 - 117] based on the optical Fourier transform of brightness pictures.

Planar modifications of the method include Particle Image Velocimetry (PIV), Micro Particle Image Velocimetry (Micro PIV), Particle Tracking Velocimetry (PTV), etc.

Volumetric methods for studying the kinematic structure of a flow include Stereo Particle Image Velocimetry (Stereo PIV), Tomographic Particle Image Velocimetry (Tomographic PIV), etc.

The PTV method is almost identical to the PIV method. The PTV method also results in instantaneous two-component velocity fields. But unlike the PIV method, the velocity vector is measured by the movements of individual tracers in the flow, not a group. The PTV method is used when the density of particle images is very low. Image processing is also performed using correlation algorithms.

One of the most important advantages of the method is the absence of a significant disturbing effect on the flow. The limitations of PIV include the finite size of the tracer particles, as a result of which the tracers do not always accurately follow the flow. This is especially true for areas of strong gradients and discontinuities. The size of the particles used limits the size of the elementary region, 
while the use of smaller particles leads to the effect of Brownian motion on their position. Tracers for gaseous media can be liquid droplets with a size of 1-100 microns; as a rule, various natural and synthetic oils are used. For high-speed streams, solid particles are used, most often from titanium oxide and aluminum. They can be smaller (on the order of hundreds of nanometers), and due to this, it is better to follow the flow.

The area of PIV method application, in particular, includes fundamental scientific research aimed at studying the dynamics and scales of vortex structures in liquid and gas flows [118-120]. In works [121-122] visualization by the PIV method of velocity profiles behind the blast (shock) waves formed during the explosion of the wire is demonstrated. With the help of an 8-pulse laser system, successive images of the velocity fields behind the front of a spherical blast wave were obtained. It is noted that a significant broadening of the velocity profile was recorded at the wave front, apparently due to the predominantly inertial impedance of liquid tracer particles. This effect, noted in other works, limits the applicability of the method to investigations of flow fields during explosions.

PIV imaging of supersonic flows remains a challenge due to the uneven particle density in the images. In this regard, the visualization of supersonic flows requires a particularly careful selection of tracer particles, flow seeding mechanisms, algorithms and parameters for PIV image processing.

Another issue, closely related to the previous one, is the adjustment of PIV data in flows with large velocity gradients. Due to the delay of particles, the data in such flows can differ significantly from the true ones. However, in some cases it is possible to take these errors into account and restore the true gas velocity field [123]. In [124], the PIV method was used to measure nonstationary velocity fields arising during the development of flows behind shock (blast) waves initiated by a pulsed surface sliding discharge in air. Plasma sheets (nanosecond discharges sliding across the dielectric surface) were initiated on the walls of a rectangular chamber. The distribution of flow velocities behind these waves showed that the impulse energy deposition is uniform along the discharge channels of the plasma sheet, while the integral apparent intensity of the plasma glow decreases in the direction of the channel propagation. Figure 18 shows the velocity field behind a plane shock wave (left side) and behind a cylindrical shock wave initiated by a nanosecond plasma channel.

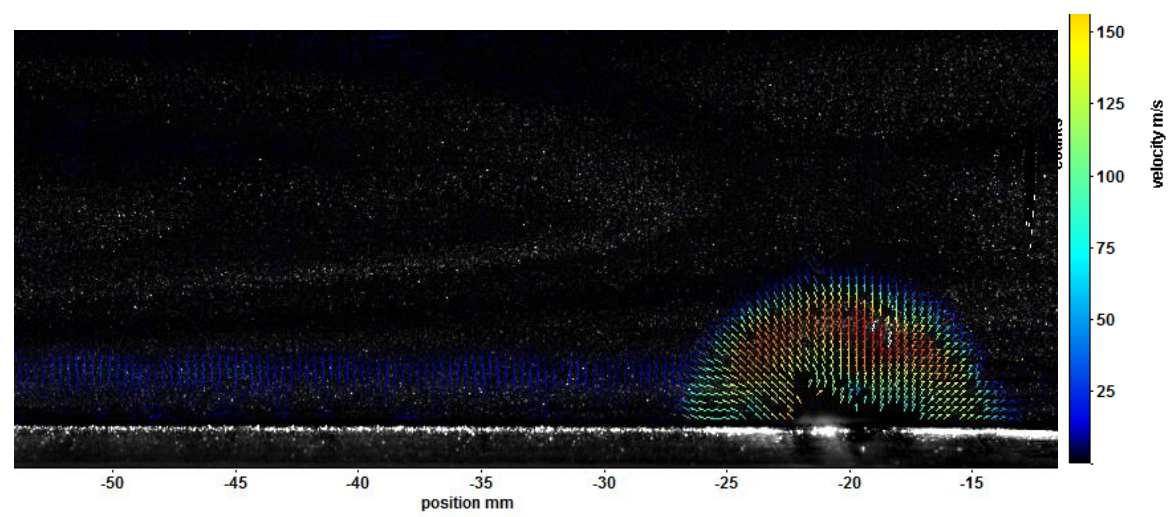

Fig. 18. Velocity field behind a shock wave initiated by a surface sliding discharge.

In recent years, the number of PIV applications for microflow researches has been increasing [125-126]. The last paper presents the results of using the micro-PIV method to visualize the flow structure in a water droplet located on a glass substrate (Fig. 19). At different moments of droplet evaporation, two-component velocity fields were obtained in different droplet height sections. 


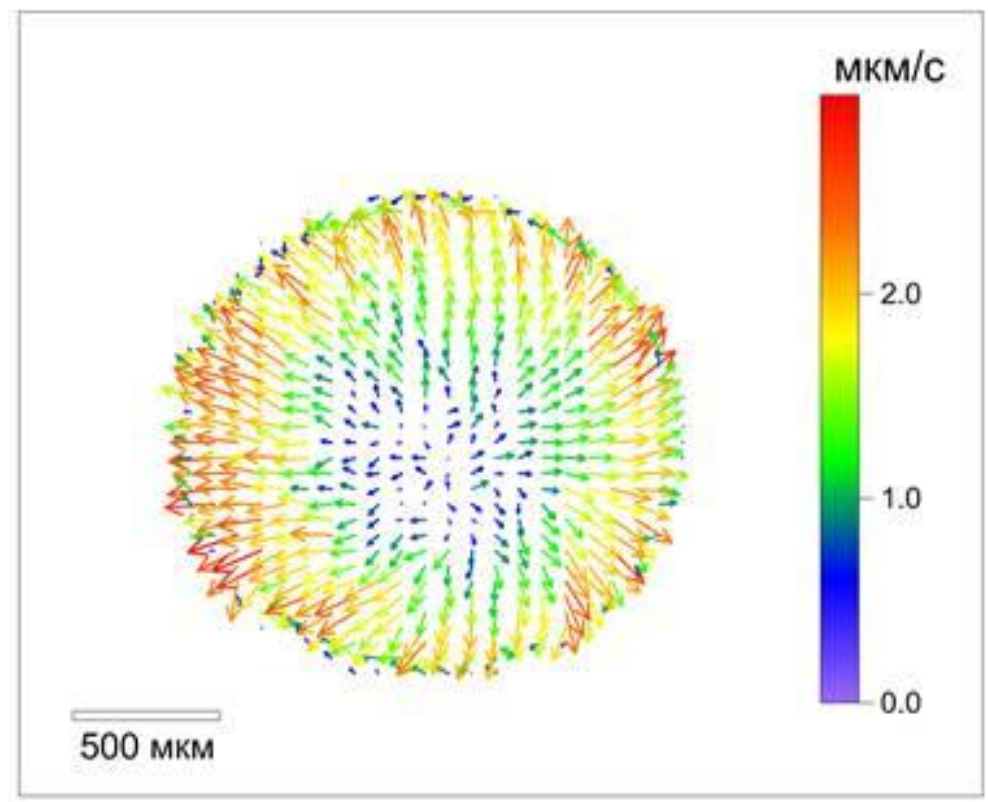

Fig. 19. Instantaneous velocity field in an evaporating droplet. Section - 45 microns from the substrate.

In [127], the PIV visualization and study of the flow developing when a shock wave exits the shock tube channel into the atmosphere was carried out (Fig. 20).

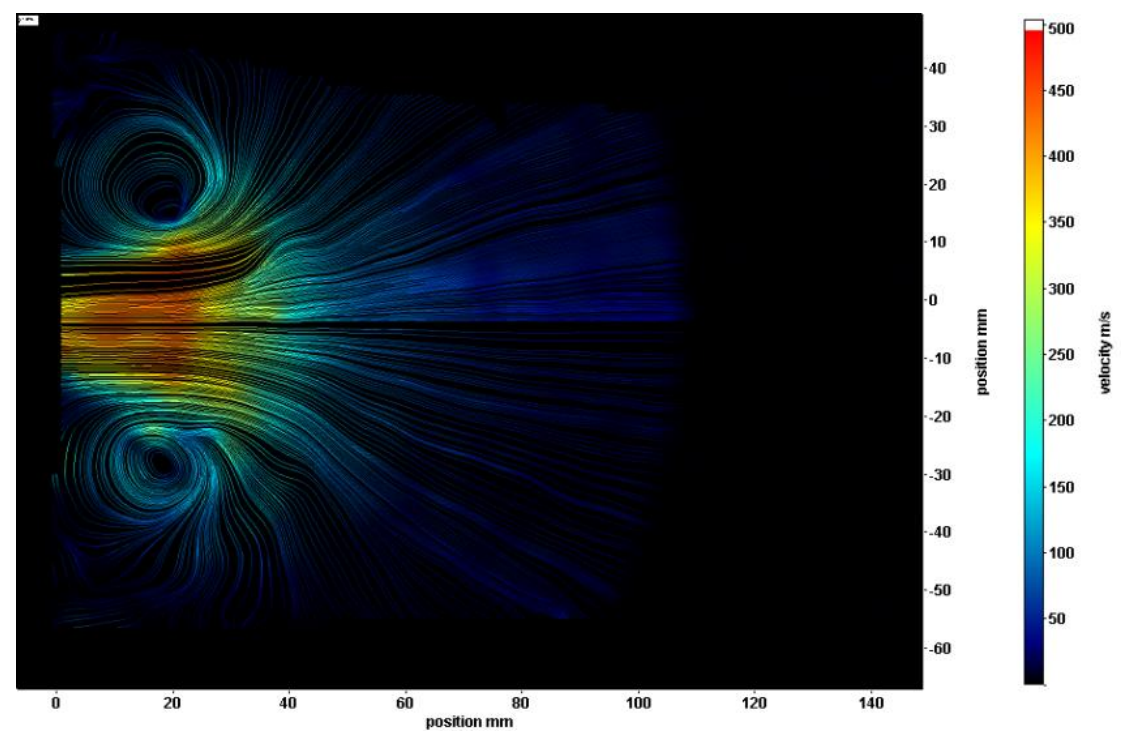

Fig. 20. Streamlines of flow behind the shock wave leaving the channel (color codes flow velocity).

In recent years, due to the spreading of cross-correlation image processing algorithms, a significant amount of papers has appeared using the seedless tracing - tracking structural elements, markers present in the flow itself. This "schlieren PIV" technique uses natural inhomogeneities and turbulent vortices in the flow as virtual "seeding particles" on which the velocity is measured [128131].

An example of visualization of boundary layer inhomogeneities in high-speed shadow image is shown in Fig. 21. The result of cross-correlation processing of such images can be used for a lower estimate of the gas velocity in the boundary layer. 


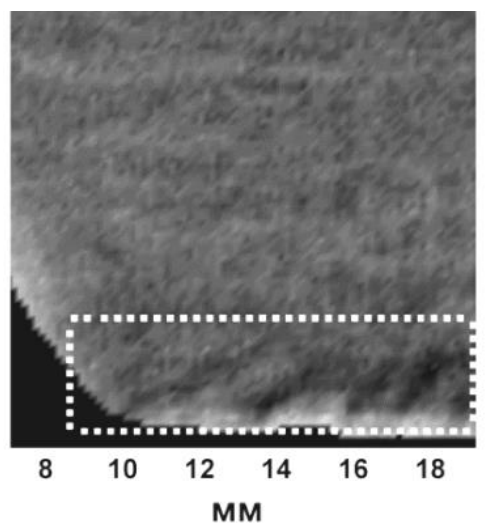

Fig. 21. A frame of high-speed shadow shooting of the flow in the test section of the shock tube.

The smoke visualization method proposed at the Kazan Scientific Center of the Russian Academy of Sciences [132] is based on digital processing of smoke visualization video recorded on a light sheet. The gas flows are seeded by aerosol generators that follows the gas flow in the same way as in PIV, but due to their higher concentration, they appear in the image as not illuminated individual particles, but smoke with a continuous intensity. The method makes it possible to use relatively primitive equipment for measuring the dynamics of two-component vector velocity fields with a frequency of about $10 \mathrm{kHz}$.

One of the seedless tracer visualization methods is a new thermal point tracing (TPT) method based on thermographic visualization of the fluid boundary layer [133]. The cross-correlation program measures the displacement of points of equal temperature (in grayscale) on two adjacent thermograms. Figure 22 shows the field of flow velocities averaged over 100 frames in the boundary layer of water based on the TPT method. We used high-speed imaging of an impact submerged non-isothermal jet with a thermal imager through a window transparent to infrared radiation. In the center is the area of laminar flow.

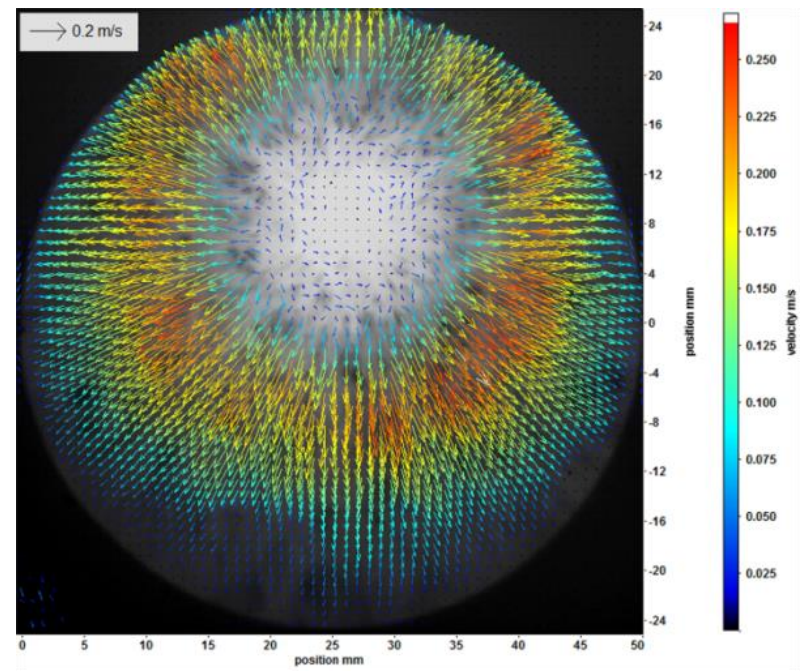

Fig. 22. Method of seedless tracing by thermal points. Velocity field in the boundary layer of an impact submerged jet.

\section{Surface flow visualization methods}

Hydrodynamic flow on a streamlined solid surface can be visualized using specially developed methods. They make it possible to detect areas of transition of a laminar boundary layer to a turbulent one, visualize zones of flow separation, areas of intersection of shock waves with the surface, distribution of thermophysical parameters over the surface, etc. In the transitional region of the flow (dynamic boundary layer), the velocity changes from zero on the wall to some final value 
in the external flow. It is also possible to distinguish a thermal (temperature) boundary layer formed in the case of a mismatch between the surface temperature and the gas temperature. When a chemical reaction proceeds on the wall or during injection, a concentration (diffusion) boundary layer is formed. The heat flow from the gas to the model can be calculated by measuring the heating rate of the model surface with known thermophysical properties.

Optical panoramic methods differ in the way they convert the surface temperature into an optical signal: fluorescent temperature converters, liquid crystals, thermal imaging. The use of special methods for visualizing the surface flow makes it possible to obtain pictures created by the distribution of velocity, temperature, pressure, and wall shear stresses in the boundary layer. The visualization of the surface flow is carried out, as a rule, as follows: a special paint, liquid film or other coating is applied to the streamlined surface of the model, which responds to the local flow parameters. Then the pattern created by the distribution of pressure, temperature, velocity, shear stresses on this surface is recorded. Coatings used to visualize surface flow, are the following: liquid crystals, coatings for infrared thermography, coatings that are sensitive to pressure (barotropic coatings, PSP) and to temperature (thermal indicator paints TSP), oil coatings, viscous oil, some special coatings, used in special cases. The methods provide both quantitative and qualitative images of the flowfields parameter on the model surface without introducing disturbances into the flow (non-contact diagnostic methods). The resulting image (film) of the flow on the surface is recorded digitally during the experiment (or after it). The problem of restoring the visualizing properties of the coating during the experiment is essential; special methods are used to solve it.

The ability to measure the light intensity and frequency has opened the way for the development of methods in which the light intensity is related to the parameter being measured (pressure, temperature, frictional stress, etc.). First of all, we focus on the methods of luminescent pressure and temperature converters.

In the late 70s, the attention of TsAGI specialists (Central Aerohydrodynamic Institute, Zhukovsky) was attracted by the work of I. Zakharov, associate professor of the Leningrad Technological Institute, who investigated the quenching of the luminescence of organic dyes (luminophores) by oxygen molecules. They used this phenomenon to measure the distribution of air pressure (which contains oxygen) on the model surface in wind tunnels. Scientists patented the idea itself in 1980, and the first pressure-sensitive coating that implements it (a layer of silica gel with organic phosphor molecules adsorbed in it) - in 1981. This coating - the authors called it a baro-indicators - turned out to be imperfect, but with its help it was already possible to see the pressure field. By the end of 1989, several different types of pressure-sensitive coatings had been developed (called luminescent pressure transducers).

The use of the phenomenon of luminescence in optical methods has the advantage over the use of scattered and reflected light that, due to the spectral shift and time delay, it makes it possible to detune from the incident (exciting) light and, thereby, increase the measuring accuracy of the intensity of light carrying useful information.

Today, the determination of the pressure distribution on a streamlined surface using coatings that are sensitive to local pressure (PSP - Pressure Sensitive Paint) is one of the main modern panoramic non-contact methods for diagnosing surface flows in thermal physics.

To visualize the pressure distribution, the surface of the model is covered with several special layers of paint; one of the layers contains a fluorescent substance with optical activity depending on the partial pressure of oxygen. The physical phenomenon underlying the visualization of pressure fields is the quenching of the luminescence of organic dyes by atmospheric oxygen. Excited by light of the appropriate wavelength, the phosphor can emit light (luminescence) or lose energy by transferring it to oxygen molecules (luminescence quenching). The share of lost energy is proportional to the oxygen concentration in the polymer and its mobility. According to Henry's law, the oxygen concentration in the polymer layer is directly proportional to its partial pressure above the polymer surface. Thus, the quantum yield of luminescence is inversely proportional to the partial pressure of oxygen. If the oxygen concentration in the air is constant, then the luminescence quenching effect can be used to measure the air pressure. A luminescent temperature 
converter (TSP) is a coating that, under the action of exciting radiation, luminesces with an intensity that depends on its temperature.

The first publications on the use of the method were published by the employees of TsAGI [134]. Similar foreign works appeared only 4-5 years later. In [135] a polymer was investigated in a laboratory shock tube, and in [136], the results of measurements of pressure and temperature in a hypersonic shock tube at the University of Kolspan are presented. The most complete reviews on the topic are presented in the monograph [137] and in the book [138]. This new edition describes pressure and temperature sensitive paints (PSP and TSP) for panoramic surface pressure and temperature measurements in aerodynamics and fluid mechanics. The book includes the latest achievements in the field of paint formulations, the results of stationary and non-stationary measurements in various aerodynamic installations, including supersonic and hypersonic wind tunnels, are presented. Technical aspects including calibration, lighting, image processing, - are described. The measurement uncertainty of PSP and TSP (Temperature Sensitive Paints) are discussed.

The papers [139-141] describe methods and results of measuring pressure and temperature fields and visualizing surface flow lines and shear stresses. Figure 23 shows the pressure field on the surface of the model, obtained using luminescent pressure transducers.

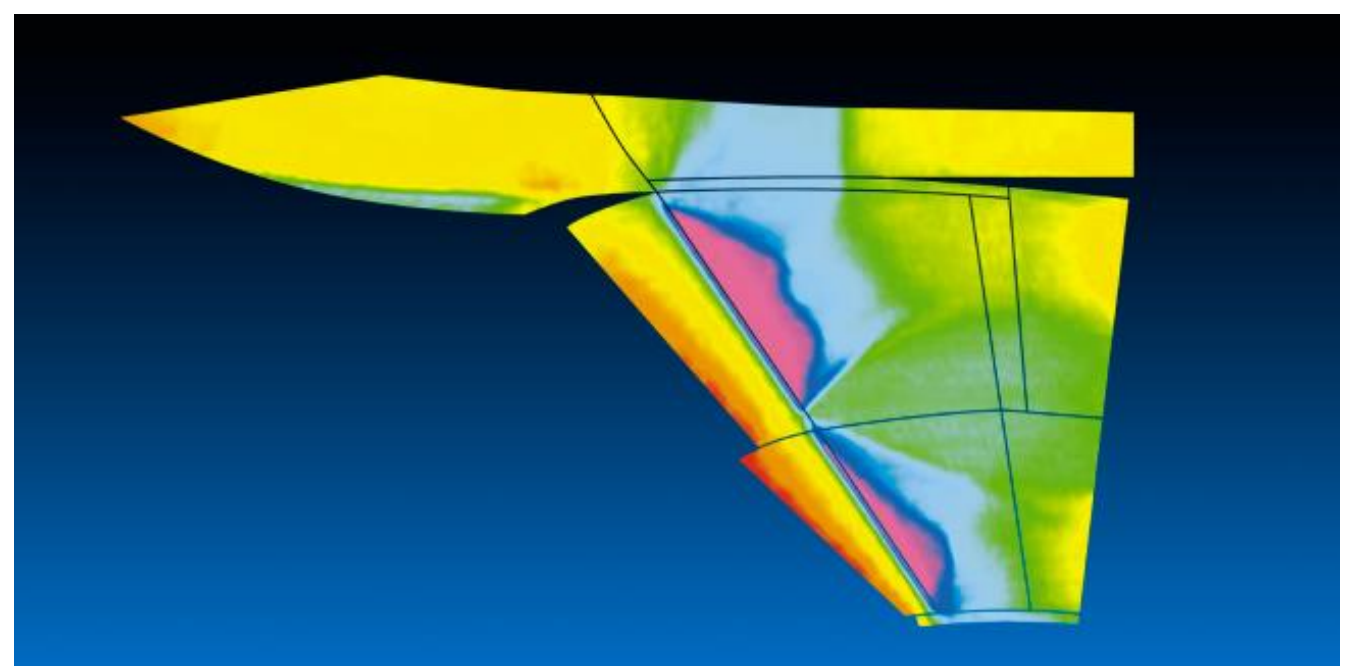

Fig. 23. Pressure distribution on the plate.

(http://www.tsagi.ru/research/measurements/lpd2.jpg)

Surface streamlines or the direction of flow at the surface of the model can be visualized by applying an oil film to the surface. Optically contrasting (luminescent) solid particles are added to the viscous oil film, and at least two distributions of these particles are recorded on the surface of the model in the flow at a given time interval using a digital camera. Processing these images using cross-correlation analysis (similar to how it is done in the PIV method) gives the particle displacement vectors. To calculate the shear stress field, it is also necessary to know the thickness of the oil film and the oil dynamic viscosity at each point of the model surface $[143,142]$. The method has become widespread in the study of separated flows. Figure 24 shows a picture of the streamlines visualization and friction stress obtained by the method of oil coatings. 


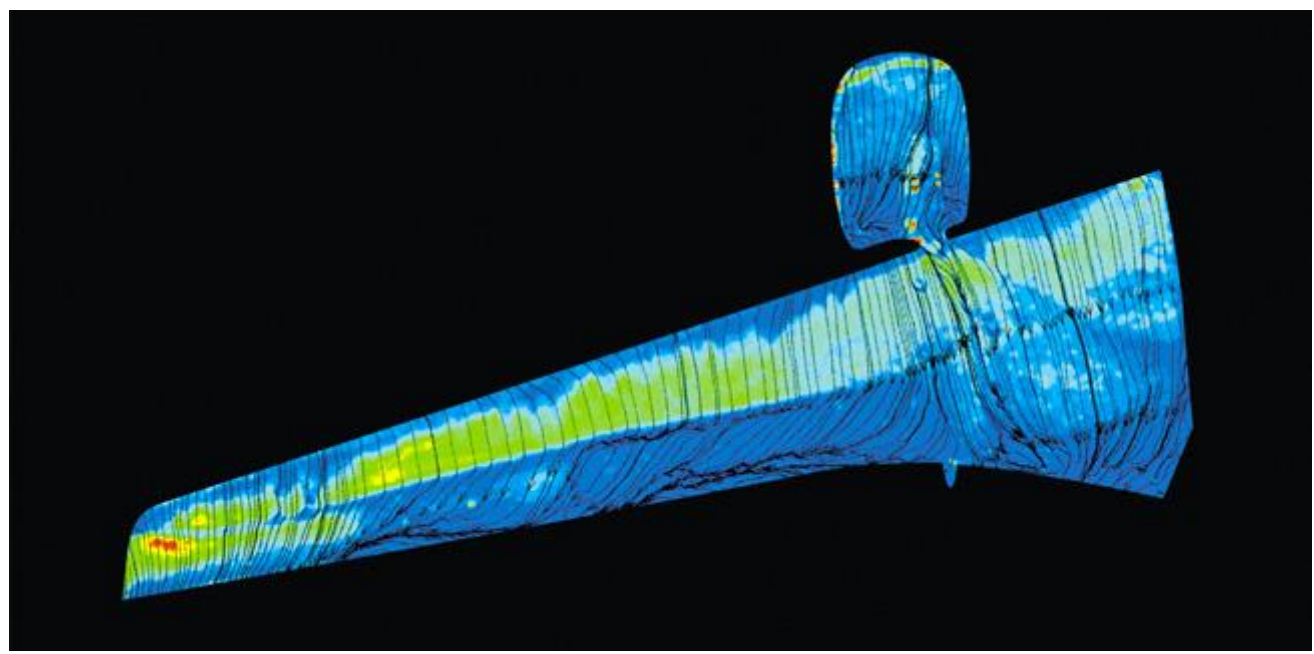

Fig. 24. Thick oil method. Wing flow of a passenger aircraft.

http://www.tsagi.ru/research/measurements/ofi/measurements_maslo.jpg

Surface interferometry in oil films is a method of measuring the resistance of the aircraft skin in flow. A layer of transparent oil applied to an air-flowed surface becomes thinner in the region where shear stress occurs. Local change in layer thickness can be measured using the interference phenomenon in thin films. Interference occurs when a surface covered with an oil film of varying thickness is illuminated by laser radiation. Interference in thin films occurs due to the interaction of light reflected from the air-oil interface and the oil-solid interface. To obtain quantitative information about the resistance of the skin, the value of the displacement of the strips is measured.

A simple but very effective method for visualizing the structure of the detonation wave front is used in combustion gas dynamics to analyze the structure of the detonation front [143]. The existence of overcompressed and undercompressed sections on the plane shock front of the detonation wave leads to the appearance of inhomogeneities. Periodic inhomogeneities in the detonation front in the form of lines of double and triple intersection of wave sections leave traces on a smoked glass plate placed at the end of the tube. This phenomenon is still used today to study the structure of the detonation front and the scale of the front inhomogeneity.

Liquid crystal coatings are used in both air and water currents. High spatial resolution, short response times, multicolor, reversibility, and relatively low cost allow using liquid crystals to visualize the temperature and shear stress distribution on the model surface, streamlines in spatial flows in a wide range of flow regimes [144]. Thermosensitive liquid crystals change color with temperature. Outside the temperature range, they become transparent on a black surface. As the temperature rises, the color changes and the border of the color area indicates the position of the isotherm on the surface. The range of temperatures measured using liquid crystal coatings is approximately from $-40^{\circ}$ to $280^{\circ} \mathrm{C}$. However, in one experiment, a range of several degrees is visualized, which means, in fact, the visualization of one isotherm with a wide range of temperatures on the surface.

\section{Visualization of CFD data: imitation of the results of a panoramic experiment}

A characteristic feature of the modern stage of visualization of thermophysical flows is the rapid convergence of numerical and experimental panoramic images of flowfields. This convergence became possible due to the introduction of digital technologies in the methods of flows registration and analysis on the one hand, and the growth of the possibilities of flows numerical modeling on the other hand. On the basis of comparison numerical calculations with the data of experimental flows visualization, the verification of models and algorithms of CFD is carried out. On the other hand, the results of the experiments are interpreted and refined based on the data of the numerical experiment data. Figure 25 shows an instantaneous image of the unsteady process of a plane shock wave diffraction in a 48x24 mm rectangular channel over a rectangular obstacle on the channel 
wall. Image was obtained by the method of flow visualization by a pulsed volume discharge. The experimental image is combined with a gas-dynamic calculation of a two-dimensional flow (density isolines, Navier-Stokes equations). The calculation helps to decode the details of the experimental image.

Numerical simulation data are visualized, if possible, in the form of experimental results simulation (numerical interferograms, pseudo-shadow images, luminescent coatings, PIV, BOS, etc.).

Today, most of the results of computational studies of supersonic gas-dynamic processes are presented in the form of numerical shadow pictures. This occurs most effectively in structured flows - with discontinuities, distinct inhomogeneities, and vortex structures.

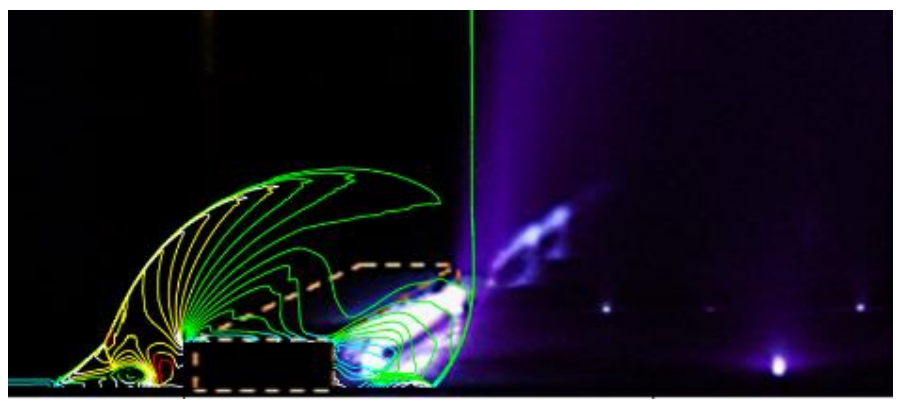

Fig. 25. Discharge glow and calculated density field in a channel with a step during the shock wave diffraction.

Figure 26 presents the calculated and experimental images of the density fields of a complex gas-dynamic quasi-two-dimensional flow in a shock tube after the shock wave interaction with a pulsed volume discharge with preionization from plasma electrodes. Comparison of experimental shadow images with the results of CDF images based on the Euler and Navier - Stokes equations made it possible to calculate the value of the energy input to the flow by solving the inverse problem [145-147].

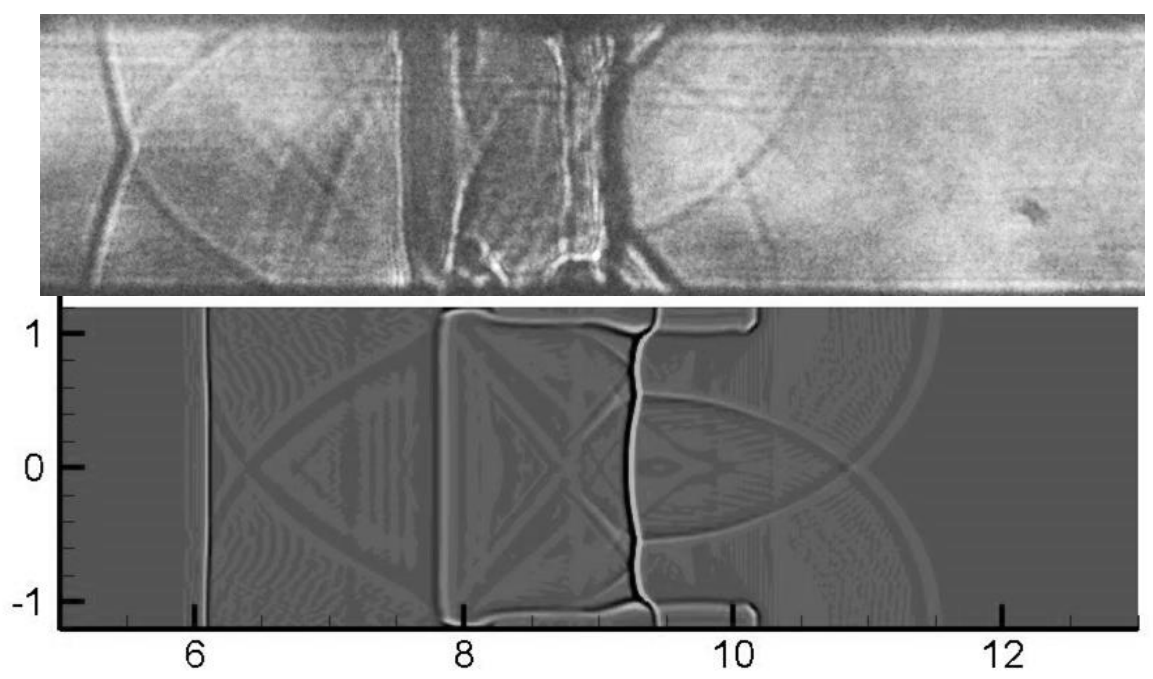

Fig. 26. Comparison of calculated and experimental shadow images.

In the 9oth the first computerized interferograms of two-dimensional flows were published $[148,149]$.

In the book [20], approaches are considered and numerous examples are given in the visual representation of a number of problems' solutions in gas dynamics, associated with calculations of inviscid and viscous compressible gas flows containing weak and strong gas-dynamic discontinuities. The data of supersonic jet visualization and separated flows obtained in experimental and numerical studies are presented. 


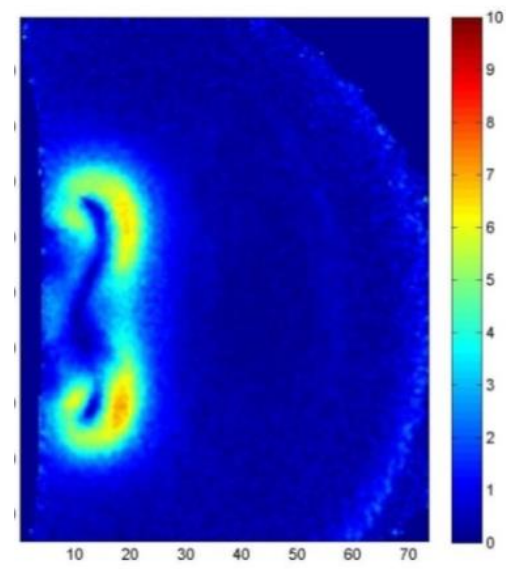

a

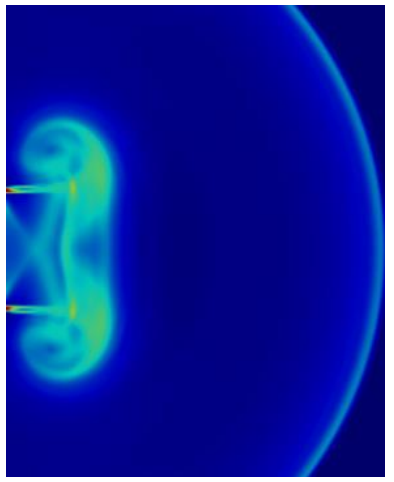

б

Fig. 27. Supersonic two-dimensional flow with a shock wave and co-flow. BOS method (displacement field) (a) and numerical simulation (b).

Figure 27 shows images of a supersonic flow after the shock wave exit from a rectangular channel, obtained by the BOS method (on the left) and numerical simulation of the corresponding flow (MSU). Figure 28 shows the result of visualization of the numerical simulation of the same flow - image of the velocity field - simulation of the digital tracing method (PIV).

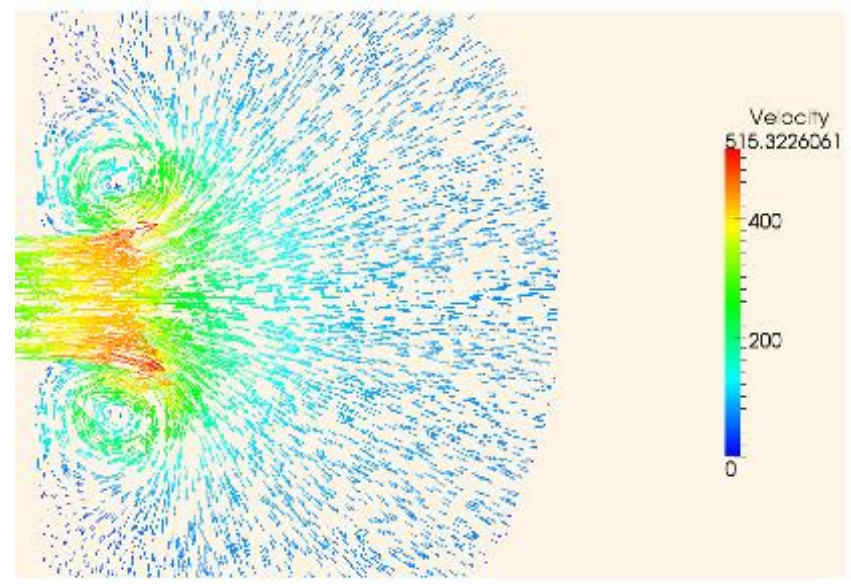

Fig. 28. Numerical simulation - the velocity field of a supersonic flow with a shock wave.

\section{Acknowledgment}

The reported study was funded by RFBR, project number 20-17-50081.

\section{References}

1. Mach, E., Salcher, P. "Photographische Fixirung der durch Projectile in der Luft eingeleiteten Vorgänge". Sitzungsber. Kaiserl. Akad. Wiss., Wien, Math.-Naturwiss. Cl. (in German). 95 (Abt. II): 764-780. 1887. Doi 10.1002/andp.18872681008.

2. Etienne-Jules Marey, "Des mouvements de l'air lorsqu'il rencontre des surfaces de differentes formes," Comptes Rendus des Seances de l'Acad 'emie des Sciences 131 (July 16, 1900): $160-63$.

3. Van Dyke, M. An album of fluid motion. Stanford, CA: Parabolic Press. 1982.

4. Yang W.J. (ed.) Handbook of Flow Visualization // NY.: Hemisphere Publishing Corporation. 1989.

5. $\quad$ Merzkich W. Flow Visualization // 2nd edition. NY.: Academic Press. 1987.

6. Mishin G.I. Optical research methods in a ballistic experiment. // L.: Nauka. 1979. 
7. Klimkin V.F., Papyrin A.N., Soloukhin R.N. Optical methods of registration of fast processes // N .: Nauka. 1980.

8. Glotov G.F., Maikapar. Aerothermodynamics of aircraft in photographs. 2003, TsAGI, Zhukovsky.

9. Panigrahi P. K., Muralidhar K., Schlieren and Shadowgraph Methods in Heat and Mass Transfer Springer, 2012.

10. Settles G.S. Schlieren and Shadowgraph Techniques. Visualizing Phenomena in Transparent Media Springer. 2001.

11. Ronald J. Adrian, Jerry Westerweel, Particle Image Velocimetry (Cambridge Aerospace), 2010.

12. Dubnischev Yu. N., Arbuzov V. A., Belousov P. P., Belousov P. Ya. Optical methods of investigation of flows. Novosibirsk: Siberian University Publishing House, 2003, 418 pp.

13. Belozerov A.F. Optical methods for visualization of streams. Kazan: publishing house KSTU, 2007, 747 pp.

14. Bazylev N.B., Fomin N.A.Quantitative visualization of currents based on speckle technologies Minsk: Belaruskaya Navuka, 2016.

15. V.P. Vavilov Infrared thermography and thermal control. 2nd edition, add. M. Publishing House Spectrum. 2009.S. 544 .

16. Modern optical methods for the study of flows / ed. B.S.Rikevichyus. - M .: Overlay, 2011.

17. Bilsky, A. V. Evolution and recent trends of particle image velocimetry for an aerodynamic experiment (review) / A. V. Bilsky, O. A. Gobyzov, D. M. Markovich // Thermophysics and Aeromechanics. - 2020. - Vol. 27. - No 1.

18. Pimshtein, V.G. Aeroacoustic interactions in turbulent jets / Moscow, 2010. - 88 c. ISBN 9785922112321.

19. Bolshukhin MA et al. Actual problems of development of an experimental base for verification of CFD codes when used in nuclear power. Proceedings of NSTU im. R.E. Alekseeva, 2013, T 2 (99) pp 117-125.

20. Volkov K.N., "Visualization of data of physical and mathematical modeling in gas dynamics" Moscow: Fizmatlit, 2018. - 356 p.

21. E. Bondarev, VA Galaktionov, VM Chechetkin, "Analysis of the development of concepts and methods of visual data representation in problems of computational physics", Zh. Vychisl. mat. and mat. Fiz., 51: 4 (2011), 669-683.

22. Krehl P., Engemann S. August Toepler // Shock Waves. 1995. V. 5. Is. 1-2. P. 1-18.

23. Litvinenko, Y.A., Grek, G.R., Kozlov, V.V., Litvinenko, M.V., Shmakov, A.G. Diffusion Combustion of a Round Hydrogen Microjet at Sub- and Supersonic Jet Velocity. Doklady Physics, V. 65, I9, 2020, P.312-316

24. Zudov V.N., Tretyakov P.K., Tupikin A.V. Ignition and stabilization by the optical discharge of homogeneous burning in high-speed jet, 2016, Scientific Visualization, V. 8, no. 2, 24 $-36 \mathrm{pp}$.

25. Vasiliev L.A. Shadow methods // Nauka. 1968.

26. Kleine H., Hiraki K., Maruyama H. et al. High-speed time-resolved color schlieren visualization of shock wave phenomena // Shock Waves. 2005. V. 14. Is. 5-6. P. 333-341.

27. Inshakov S.I., Rodionov A.Yu., Shirin A.S., Shekhtman V.N. Eleventh International Scientific and Technical Conference "Optical Methods for Studying Flows", Moscow, June 27 - 30, 2011 interferometer for simultaneous registration of two interferograms with an orthogonal shear direction.

28. Bukin V.V., Garnov S.V., Malyutin A.A., Strelkov V.V. Interferometric diagnostics of femtosecond laser microplasma in gases // Trudy instituta obshchey fiziki im. A.M. Prokhorova, 2011, Volume 67, p. 3.

29. Dubnishchev Yu.N., Arbuzov V.A., Arbuzov E.V., Berdnikov V.S., Kislytsin S.A., Melekhina O.S. Optical diagnostics of convective structures induced by non-stationary boundary conditions in a vertical water layer // Scientific Visualization, 2018, V. 10, no. 4, 134 - 144 pp. 
30. Wei W., Lia X., Wu J., Yang Z., Jia S., Qiu A. Interferometric and schlieren characterization of the plasmas and shock wave dynamics during laser-triggered discharge in atmospheric air // Physics of Plasmas, Vol. 21, No. 8, 2014.

31. Hargather J., Settles S. A review of recent developments in schlieren and shadowgraph techniques // Meas. Sci. Technol 2017. V. 28. N. 4.

32. A.A. Kandaurov, D.A. Sergeev, O.S. Ermakova, Yu.I. Troitskaya. Investigation of the mechanisms of spray generation induced by wind-wave interactionusing shadow technique. Scientific Visualization V.9 no. 3, 103 - 107 pp.

33. Alferov V.I., Bushmin A.S. Electric discharge in a supersonic air flow // ZhETF, 1963, V. 44, V. 6, pp. 1775-1779.

34. Alferov, V.I. Kalachev, B.V., Visualization of supersonic flows by means of a prebreakdown discharge, Journal of Applied Mechanics and Technical Physics, 9(4), pp. 468-470.

35. Alferov V.I. Investigation of the structure of a high-power electric discharge in a highspeed air flow // Izvestiya. AN SSSR, MZhG, 2004, No. 6, pp. 163-175, 1968.

36. Alferov V.I. On the question of determining the flux density in the visualization of vortex bundles by the method of high-voltage discharge // Tr. TsAGI. 1972. Issue. 1421.S. 13-2.

37. Alferov V.I., Dmitriev L.M. Electric discharge in a gas flow in the presence of density gradients // TVT, 1985, T. 23, No. 4, pp. 677-682.

38. Nishio M., Sezaki S., Nakamura H. Visualization of Flow Structure Around a Hypersonic Re-entry Capsule Using the Electrical Discharge Method // J. of Visualization, Vol.7, No.2, 2004.

39. Nishio M., Nakamura H., Sezaki Sh., Manabe K. Flowfield Around Space Plane Traveling at Mach 10 (Comparison of Visualization and Calculation) // The 10th International Symposium on Flow Visualization, Aug.26-29, 2002, Kyoto, Japan.

40. Jagadeesh G., Srinivasa Rao B.R., Nagashetty K., Reddy N.M., Reddy K.P.J. Electrical discharge technique for three-dimensional flow field visualisation in hypersonic shock tunnel // J. Flow Visualisation and Image Processing, Vol. 4, No. 1, pp. 51-57, 1997.

41. Jagadeesh G., Srinivasa Rao B.R., Nagashetty K., Reddy K.P.J., Viren M. Visualization studies around spiked blunt cones using electrical discharge technique at Mach 5.75 // The 1oth International Symposium on Flow Visualization, Aug. 26-29, 2002, Kyoto, Japan.

42. Chen X., Sha X., Wen S., Lu H.B., Ji F. Visualization of three dimension shock wave in hypersonic gun tunnel using electric discharge. Proceedings 18th International Symposium on Flow Visualization. Zurich, Switzerland, 2018.

43. Ieshkin A.E, Danilov A.V, Chernysh V.S, Ivanov I.E, Znamenskaya I.A. Visualization of supersonic flows with bow shock using transversal discharges. Journal of Visualization, Vol. 22, pp. 741-750, 2019.

44. Znamenskaya I. A., Kuli-zade T. A., Kulikov V. N., Perminov S. P. Transonic 3d nonstationary flow visualization using pulse transversal discharge // Journal of Flow Visualization and Image Processing. - 2011. - Vol. 18, no. 3. - P. 214-224.

45. I. A. Znamenskaya, T. A. Kuli-zade, "Visualization of toroidal vortex instability by the impulse volume discharge", Dokl. Akad. Nauk, 348:5 1996, 617-619.

46. Znamenskaya I. A., Ivanov I. E., Kryukov I. A., Kuli-Zade T. A. Pulsed volume discharge with preionization in a two-dimensional gas-dynamic flow// JETP, Vol. 95, No. 6, p. 1033, December 2002

47. Znamenskaya I. A., Tatarenkova D. I., Kulizade T. A. Nanosecond ionization of an area of flowing around a rectangular ledge by a high-speed flow // Technical Physics Letters. - 2020. Vol. 46. - P. 5-7.

48. Li G. et al. Image Processing Techniques for Shock Wave Detection and Tracking in High Speed Schlieren and Shadowgraph Systems. // Journal of Physics: Conference Series, vol. 1215, 2019.

49. Edla D. et al. Advances in Machine Learning and Data Science: Recent Achievements and Research Directives. Springer, 2018. 
50. Ye S. et al. A flow feature detection method for modeling pressure distribution around a cylinder in non-uniform flows by using a convolutional neural network. Scientific Reports, vol. 10, 2020.

51. Canny J. A. Computational Approach to Edge Detection. IEEE Transactions on Pattern Analysis and Machine Intelligence, vol. PAMI-8(6), p. 679-698, 1986.

52. Srisha Rao M., Jagadeesh G. Visualization and Image Processing of Compressible Flow in a Supersonic Gaseous Ejector. // Journal of the Indian Institute of Science, vol. 93, no. 1, 2013.

53. Fujimoto T.R., Kawasaki T., Kitamura K. Canny-Edge-Detection/Rankine-HugoniotConditions Unified Shock Sensor for Inviscid and Viscous Flows // J. Comput. Phys., vol. 396, pp. 264-279, 2019.

54. Brunton S. L. et al. Machine Learning for Fluid Mechanics. Annual Review of Fluid Mechanics, vol. 52, pp. 477-508, 2020.

55. Dehghan Manshadi M. et al. Speed Detection in Wind-tunnels by Processing Schlieren Images. IJE TRANSACTIONS A: Basics, vol. 29, no. 7, pp. 962-967, 2016.

56. Colvert B. et al. Classifying vortex wakes using neural networks. Bioinspiration \& Biomimetics, vol 13, no. 2, 2018.

57. Harel R., Rusanovsky M., Fridman Y., Shimony A., Oren G. Complete Deep ComputerVision Methodology for Investigating Hydrodynamic Instabilities. High Performance Computing. ISC High Performance 2020. Lecture Notes in Computer Science, Vol 12321, p. 61-80, 2020.

58. Ott C., Pivot C., Dubois P., Gallas Q., Delva J., Lippert M., Keirsbulck L. Pulsed jet phaseaveraged flow field estimation based on neural network approach. Experiments in Fluids, Vol. 62, No. 79, 2021.

59. Kutz J. Deep learning in fluid dynamics. // Journal of Fluid Mechanics, Vol. 814, pp 1-4, 2017.

6o. Znamenskaya I. A., Doroshchenko I.A. Edge detection and machine learning for automatic flow structures detection and tracking on schlieren and shadowgraph images // Journal of Flow Visualization and Image Processing. - 2021. - Vol. 28, no. 4. - P. 1-26.

61. I. A. Znamenskaya, I. A. Doroshchenko, N. N. Sysoev, and D. I. Tatarenkova. Results of Quantitative Analysis of High-Speed Shadowgraphy of Shock Tube Flows Using Machine Vision and Machine Learning. Doklady Rossiiskoi Akademii Nauk. Fizika, Tekhnicheskie Nauki, Vol. 497, pp. 16-20, 2021.

62. Znamenskaya, I.A. et al. Edge detection and machine learning approach to identify flow structures on schlieren and shadowgraph images. Proceedings of the 3oth International Conference on Computer Graphics and Machine Vision. CEUR Workshop Proceedings, vol. 2744, pp. 1-14, 2020.

63. V.P. Vavilov Infrared thermography and thermal control. 2nd edition, add. M. Publishing House Spectrum. 2013. - P. 544.

64. Chernorizov A., Isaychev S., Znamenskaya I., Koroteyeva E., Khakhalin A., Shishakov V. Remote Detection of Human Emotional States by Facial Areas // International Journal of Psychophysiology. - 2018. - 131. - S. 85.

65. Berlovskaya E.E., Isaychev S.A., Chernorizov A. M., Ozheredov I.A., Adamovich T.V., Isaychev E.S., Cherkasova O.P., Makurenkov A.M., Shkurinov A.P., Varaksin A.N., Gatilov S.B., Kurenkov N.I., Manaenkov A.E. Diagnosing Human Psychoemotional States by Combining Psychological and Psychophysiological Methods with Measurements of Infrared and $\mathrm{THz}$ Radiation from Face Areas // Psychology in Russia: State of the Art. - 2020. - 13. - №. 2. - P. 6483.

66. Carlomagno G.M., Cardone G. Infrared thermography for convective heat transfer measurements // Experiments in fluids. - 2010. - 49. - №. 6. - P. 1187-1218.

67. Leontiev A.I., Kiselev N.A., Burtsev S.A., Strongin M M., Vinogradov Y.A. Experimental investigation of heat transfer and drag on surfaces with spherical dimples // Experimental Thermal and Fluid Science. - 2016. - 79. - P. 74-84. 
68. Kiselev N.A., Leontiev A.I., Vinogradov Yu.A. et al. Effect of large-scale vortex induced by a cylinder on the drag and heat transfer coefficients of smooth and dimpled surfaces // International Journal of Thermal Sciences. - 2019. - 136. - P. 396-409.

69. Thomann H., Frisk B. Measurement of heat transfer with an infrared camera //International Journal of Heat and Mass Transfer. - 1968. - 11. - №. 5. - P. 819-826.

70. Bazovkin V.M., Kovchavtsev A.P., Kuryshev G.L., Maslov A.A., Mironov S.G., Khotyanovskiy D.V., Tsarenko A.V., Tsyryulnikov I.S. Numerical and experimental investigation of hypersonic flow around a two-dimensional compression angle. // Journal Vestnik NSU. Series Physics. 2007. Volume 2, No. 1. P. 3-9.

71. Simon B., Filius A., Tropea C., Grundmann S. // Experiments in Fluids. - 2016. - 57. №. 5. - P. 93.

72. Raffel M., Merz C.B. Differential Infrared Thermography for Unsteady Boundary-Layer Transition Measurements // AIAA journal. - 2014. - 52. - №. 9. - P. 2090-2093.

73. Richter K., Schulein E. Boundary-layer transition measurements on hovering helicopter rotors by infrared thermography // Experiments in fluids. - 2014. - 55. - №. 7. - P. 1755 .

74. Banks D.W., Frederick M.A., Tracy R.R., Matisheck J.R., Vanecek N.D. In-flight boundary-layer transition on a large flat plate at supersonic speeds. 15th International Symposium on Flow Visualization ISFV15 - Minsk / Belarus - 2012 0-62.

75. V. S. Berdnikov, V. A. Grishkov, K. Y. Kovalevskii, V. A. Markov. Thermal Imaging Studies of the Laminar-Turbulent Transition in the Rayleigh-Benard Convection. Avtometriya, 2012, n.3, P. 111-120.

76. Ivanitskii G R, Deev A A, Khizhnyak E P "Water surface structures observed using infrared imaging" Phys. Usp. 2005, V 48, P. 1151-1159.

77. Hetsroni G., Mewes D., Enke C., Gurevich M. et al. Heat transfer to two-phase flow in inclined tubes // International Journal of Multiphase Flow. - 2003. - 29. - №. 2. - P. 173-194.

78. Error! Reference source not found.

79. Carlomagno G.M., Ianiro A. Thermo-fluid-dynamics of submerged jets impinging at short nozzle-to-plate distance: A review // Experimental thermal and fluid science. - 2014. - 58. - P. 1535 .

80. Judd K.P., Smith G.B., Handler R.A., Sisodia A. The thermal signature of a low Reynolds number submerged turbulent jet impacting a free surface //Physics of Fluids. - 2008. - 20. - №. 11. -115102.

81. Kashinskii, O.N., Lobanov, P.D., Kurdyumov, A.S. et al. Experimental simulation of a liquid-metal heat-transfer fluid flow in a T-shaped mixer. Tech. Phys. 61, 783-785 (2016).

82. Zaitsev D.K., Smirnov E.M., Kolesnik E.V., Bolshukhin M.A., Budnikov A.V., Sveshnikov D.N. Computational and experimental study of temperature pulsations in a tee joint with oblique injection // Collection of reports " Problemy primeneniya i verifikatsii CFD kodov v atomnoy energetike". - 2018. - C. 104-105.

83. Bol'shov L., Pribaturin N., Kashinsky O., Lobanov P., Kurdyumov A. Experimental Study of Mixing Fluid Flows with Different Temperatures in a T-Junction // Journal of Applied Mechanics and Technical Physics. - 2020. - 61. - №. 3. - P. 368-376.

84. Nakamura H., Shiibara N., Yamada S. Quantitative measurement of spatio-temporal heat transfer to a turbulent water pipe flow // International Journal of Heat and Fluid Flow. - 2017. 63. - P. 46-55.

85. Roux S., Fenot M., Lalizel G., Brizzi L.-E., Dorignac E. Evidence of flow vortex signatures on wall fluctuating temperature using unsteady infrared thermography for an acoustically forced impinging jet // International journal of heat and fluid flow. - 2014. - 50. - P. 38-50.

86. Nakamura H. Measurements of time-space distribution of convective heat transfer to air using a thin conductive film // Fifth International Symposium on Turbulence and Shear Flow Phenomena. - Begel House Inc., 2007. - P. 1906-1914.

87. Shiibara N., Nakamura H., Yamada S. Visualization of turbulent heat transfer to a water flow in a circular pipe using high-speed infrared thermography //Journal of Flow Visualization and Image Processing. - 2013. - 20. - №. 1-2. 
88. Znamenskaya I.A., Koroteeva E.Yu., Shirshov Ya.N., Novinskaya A.M., Sysoev N.N. High speed imaging of a supersonic waterjet flow //Quantitative InfraRed Thermography Journal. 2017. - V. 14. - №. 2. - P. 185-192.

89. Bolshukhin, M.A., Znamenskaya, I.A. \& Fomichev, V.I. A method of quantitative analysis of rapid thermal processes through vessel walls under nonisothermal liquid flow. Dokl. Phys. 2015, V. 60, P. 524-527.

90. Koroteeva E., Shagiyanova A., Znamenskaya I., Sysoev N. Time-resolved thermographic analysis of the near-wall flow of a submerged impinging water jet // Experimental Thermal and Fluid Science. - 2021. - P. $110-264$.

91. Znamenskaya I., Koroteeva E., Shagiyanova A. Thermographic analysis of turbulent nonisothermal water boundary layer // Journal of Flow Visualization and Image Processing. - 2019. - Vol. 26, no. 1. - P. 49-56.

92. P.P.Khramtsov et al. Diagnostics of Density Fields in Hypersonic Flows around a Cone in a Light-Gas Gun by the Shadow Photometric Method. Tech. Phys. 64, 1424-1429 (2019).

93. Meier G., (2002). Computerized background-oriented schlieren. Exp. Fluids 33 (1), pp.181-187.

94. Dalziel S.B., Hughes G.O., Sutherland B.R., (2000). Whole-field density measurements by "synthetic schlieren." Exp. Fluids 28 (4), pp.322-335.

95. Bazylev N.B., Fomin N.A.Quantitative visualization of currents based on speckle technologies - Minsk: Belaruskaya Navuka, 2016 .-- 392 p.

96. Khramtsov P.P., Penyazkov O.G., Shatan I.N. Temperature measurements in an axisymmetric methane-air flame using Talbot images. Exp Fluids 56, 31 (2015).

97. Raffel M. Background-Oriented Schlieren (BOS) techniques. Exp Fluids 56, 60 (2015).

98. Skornyakova N.M. Background Oriented Schlieren and its applications. In the book Modern optical methods for the study of flows / ed. B.S.Rikevichyus. - M .: Overlay, 2011.S. 93-107.

99. Hazewinkel J., Maas L. R. M., Dalziel S. B. Tomographic reconstruction of internal wave patterns in a paraboloid. Experiments in Fluids, vol. 50, no. 2, pp. 247-258, Jul. 2010.

100. Kirmse T., Agocs J., Schröder A., Martinez Schramm J., Karl S., Hannemann, K., (2011). Application of particle image velocimetry and the Background-Oriented Schlieren technique in the high-enthalpy shock tunnel Göttingen. Shock Waves 21 (3), pp.233-241.

101. Glazyrin F.N., Znamenskaya I.A., Mursenkova I.V., Sysoev N.N., Jin J., (2012). Study of shock-wave flows in the channel by schlieren and background oriented schlieren methods. Optoelectron. Instrum. Data Process. 48 (3), pp.303-310.

102. Glazyrin F., Znamenskaya I., Koroteeva E., Mursenkova I., Sysoev N. Application of Background Oriented Schlieren Technique for Investigations of a Non-Stationary Flow With Shock Wave // Scientific Visualization - 2013. - V.5 - № 3-65-74 pp.

103. Tillmann W., Abdulgader M., Rademacher H., Anjami N., Hagen L., (2014). Adapting of the Background-Oriented Schlieren (BOS) Technique in the Characterization of the Flow Regimes in Thermal Spraying Processes. J. Therm. Spray Technol. 23 (1-2), pp.21-30.

104. Mizukaki T., Wakabayashi K., Matsumura T., and Nakayama K., (2014). BackgroundOriented Schlieren with natural background for quantitative visualization of open-air explosions. Shock Waves 24 (1), pp.69-78, 2014.

105. Gerasimov S.I., Trepalov N.A. Background-Oriented Schlieren method for recording of air shock waves. Scientific Visualization, 2017, 4(9), 1-12 pp.

106. Sysoev N.N., Znamenskaya I.A. New possibilities of digital technologies for image analysis during testing at proving grounds. Izvestiya Rossiyskoy akademii raketnykh i artilleriyskikh nauk. - 2020. - T. 112, No. 2. - P. 114.

107. Znamenskaya I.A., Vinnichenko N.A., Glazyrin F.N., (2012). Quantitative measurements of the density gradients on the flat shock wave by means of Background Oriented Schlieren. (ISFV, Minsk, Belarus), p. o6o.

108. Hazewinkel J., Maas L.R.M., Dalziel S.B., (2011). Tomographic reconstruction of internal wave patterns in a paraboloid. Exp. Fluids 50 (2), pp.247-258. 
109. Hayasaka K., Tagawa Y. Mobile visualization of density fields using smartphone Background-Oriented Schlieren. Exp Fluids 60, 171 (2019).

110. Hargather M.J., Settles G.S. (2012) A comparison of three quantitative schlieren techniques. Opt Lasers Eng 50(1):8-17.

111. Fisher T.B., Quinn M.K., Smith K.L. An experimental sensitivity comparison of the schlieren and Background-Oriented Schlieren techniques applied to hypersonic flow // Measurement Science and Technology, 2019, V. 30, N. 6.

112. Adrian R. J. Particle Image Velocimetry / Adrian R. J., Westerweel J. Particle Image Velocometry. Evolution and recent trends of particle image velocimetry for an aerodynamic experiment (review) // Cambridge University Press, 2010. - 586 p.

113. Raffel M., Willert C.E., Scarano F., Kähler C., Wereley S.T., Kompenhans J. 2018. Particle image velocimetry, a practical guide 3rd ed. Springer Int. Publishing. 669 p.

114. Scarano F. 2012. Tomographic PIV: principles and practice // Meas. Sci. Technol. Vol. 24, No. 1. P. 012001-1- 012001-28.

115. Grant, I. Particle image velocimetry: A review. Arch. Proc. Inst. Mech. Eng. C J. Mech. Eng. Sci. 1997, 211, 55-76.

116. N. B. Bazylev, N. A. Fomin. Quantitative visualization of currents based on speckle technologies Minsk: Belaruskaya Navuka, 2016.392p.(In Russian).

117. Adrian R. J. Twenty years of particle image velocimetry // Exp. in Fluid s. - 2005. - Vol .39. - P. 159-169.

118. Mariani R., Kontis K., (2010). Experimental studies on coaxial vortex loops. pp.126102. Physics of Fluids 22(12) .

119. Skornyakova N.M., Sychev D.G., Varaksin A.Yu., Romash M.E. (2015) Vizualization of vortex structures by particle image velocimetry. Scientific Visualization, 7 (3), pp.15-24.

120. Koroteeva E.Y., Znamenskaya I.A., Glazyrin F.N., Sysoev N.N. Numerical and experimental study of shock waves emanating from an open-ended rectangular tube // Shock Waves. - 2016. - Vol. 26, no. 3. - P. 269-277.

121. Murphy M.J., Adrian R.J., (2010). PIV space-time resolution of flow behind blast waves. Exp. Fluids 49 (1), pp.193-202.

122. Murphy M.J., Adrian, R.J., (2011). PIV through moving shocks with refracting curvature. Exp. Fluids 50 (4), pp.847-862.

123. Koroteeva E., Mursenkova I., Liao Y., Znamenskaya I. Simulating particle inertia for velocimetry measurements of a flow behind an expanding shock wave // Physics of Fluids. - 2018. - Vol. 30, no. 1. - P. 011702.

124. PIV analysis of the homogeneity of energy deposition during development of a plasma actuator channel / F. N. Glazyrin, I. A. Znamenskaya, I. V. Mursenkova et al. // Technical Physics Letters. - 2016. - Vol. 42, no. 1. - P. 63-66.

125. Bin Yang, Yuan Wang, Wen Bo He "Application of Micro-PIV on the Microscale Flow and a Modified System Based on Ordinary 2-D PIV.” Advanced Materials Research 346 (2011): 65763.

126. Yagodnitsyna A.A., Bilsky A.V., Kabov O.A. Flow visualization in evaporating droplet on a substrate by means of micro-PIV technique // Scientific Visualization, 2016, V. 8, no. 2, 53-58 pp.

127. Koroteeva E. Y., Znamenskaya I. A., Glazyrin F. N., Sysoev N. N. Numerical and experimental study of shock waves emanating from an open-ended rectangular tube // Shock Waves. - 2016. - Vol. 26, no. 3. - P. 269-277.

128. Dennis R. Jonassen, Gary S. Settles, Michael D. Tronosky, Schlieren "PIV" for turbulent flows, Optics and Lasers in Engineering, Volume 44, Issues 3-4, 2006, Pages 190-207.

129. Michael John Hargather Michael James Lawson Gary S. Settles Leonard M. Weinstein. Seedless Velocimetry Measurements by Schlieren Image Velocimetry 2011 AIAA Journal 49(3):611-620. 
130. Nematollahi O., Samsam-Khayani H., Kim K.C., Nili-Ahmadabadi M., Yoon S.Y A novel self-seeding method for particle image velocimetry measurements of subsonic and supersonic flows Scientific Reports. 2020. T. 10. № 1. C. 10834.

131. Bryan E. Schmidt, Wayne E. Page, Jeffrey A. Sutton Seedless Velocimetry in a Turbulent Jet using Schlieren Imaging and a Wavelet-based Optical Flow Method AIAA 2020-2207 Session: Advancements in Planar, Volumetric, and High-Speed Imaging Techniques.

132. Mikheev, N.I., Dushin, N.S. A method for measuring the dynamics of velocity vector fields in a turbulent flow using smoke image-visualization videos. Instrum Exp Tech 59, 882-889. 2016.

133. Koroteeva E. Y., Znamenskaya I. A., Ryazanov P. A. Velocity-field measurements in a fluid boundary layer based on high-speed thermography // Doklady Physics. - 2020. - Vol. 65, no. 3. - P. 100-102.

134. Borovoy V., Bykov A., Mosharov V., Orlov A., Radchenko V., Fonov S. Pressure Sensitive Paint Application in Shock Wind Tunnel. // 16th ICIASF Congress. - Dayton, Ohio, July 1995, ICIASF 95 record, 1995. P. 34.1-34.4.

135. Hubner J.P., Carroll B.F., Schanze K.S., Ji H.F. Pressure sensitive Paint Measurements in a Shock Tube. // Experiments in Fluids, 2000. - V. 28. - № 1. - P. 21-28.

136. Hubner J.P., Carroll B.F., Schanze K.S., Ji H.F., Holden M.S. Temperature- and PressureSensitive Paint Measurements in Short-Duration Hypersonic Flow. // AIAA Journal, 2001. - V. 39. - № 4. - P. 654-659.

137. Liu T., Sullivan J.P. Pressure and Temperature Sensitive Paints // Springer 2005, 328c.

138. Liu T., Sullivan J.P., Asai K., Klein C., Egami Y. 2nd ed. 2021. Springer.

139. Mosharov V. E. "Luminescent methods for investigating surface gas flows (Review)" Instruments and Experimental Techniques January, Volume 52, Issue 1, pp 1-12. (2009).

140. Mosharov V., Radchenko V. PSP/TSP activity in TsAGI // 15th International Symposium on Flow Visualization (ISFV15) proceedingss, Minsk, Belarus, June 25-28, 2012, CD, ISFV15-009, pp.1-4.

141. Mosharov V., Radchenko V., Tsipilev N. Particle image surface flow visualization and skin-friction measurement // 31st Congress of the International Council of the Aeronautical Sciences, ICAS 2018 : 31, Belo Horizonte, 2018.

142. Mosharov V. Ye., Radchenko V. N., Tsipilev N. S. Visualization of the flow on the surface from the images of particles: a step towards the measurement of surface friction // Modeli i metody aerodinamiki : Shestnadtsataya Mezhdunarodnaya shkola-seminar, Evpatoria, 2016- C . 118-119 TsAGI.

143. Shchelkin K.I., Troshin Ya.K. Combustion gas dynamics. M. 1963.

144. Zharkova G.M., Kovrizhina V.N., Petrov A.P., Shapoval, E.S., Mosharov V.E. and Radchenko V.N. Visualization of boundary layer transition by shear sensitive liquid crystals.// Proceedings PSFVIP-8: 2011 Moscow, Russia. No. 113. - P. 1-5.

145. Znamenskaya I. A., Koroteev D. A., Lutsky A. E. Discontinuity breakdown on shock wave interaction with nanosecond discharge // Physics of Fluids. - 2008. - Vol. 20. - P. 056101-1056101-6.

146. Chetverushkin, B.N., Znamenskaya, I.A., Lutsky, A.E. et al. Numerical Simulation of the Interaction and Evolution of Discontinuities in a Channel Based on a Compact Form of QuasiGasdynamic Equations. Math Models Comput Simul 2021, 13, P. 26-36.

147. Koroteeva E., Znamenskaya I., Orlov D , Sysoev N.Shock wave interaction with a thermal layer produced by a plasma sheet actuator // Journal of Physics D - Applied Physics. - 2017. Vol. 50, no. 8. - P. 085204 .

148. Tamura Y., Fujii K. Visualization for computational fluid dynamics and the comparison with experiments, Paper AIAA-90-3031 (1990).

149. Fursenko A. A., Sharov D. M., Timofeev E. V., Voinovich P. A. Numerical Simulation of Shock Wave Interactions with Channel Bends and Gas Nonunoformities. // Computers Fluids Vol. 21, No. 3, pp. 377-396, 1992. 